\title{
Second order discontinuous Galerkin scheme for compound natural channels with movable bed. Applications for the computation of rating curves
}

\author{
Lorenzo Minattia,*, Pina Nicoletta De Cicco ${ }^{\mathrm{b}}$, Luca Solari ${ }^{\mathrm{b}}$ \\ a CeRAfRi, Via di S.Marta 3, 50139, Firenze, Italy \\ b Civil and Environmental Engineering Department, University of Florence, Via di S.Marta 3, 50139, Firenze, Italy
}

\section{A R T I C L E I N F O}

\section{Article history:}

Available online xxx

\section{Keywords:}

Shallow water equations

Movable bed rating curves

Discontinuous Galerkin schemes

Well-balanced schemes

Stiff source term treatment

Bedload transport

\begin{abstract}
A B S T R A C T
A new higher order 1D numerical scheme for the propagation of flood waves in compound channels with a movable bed is presented. The model equations are solved by means of an ADER Discontinuous Galerkin explicit scheme which can, in principle, reach any order of space-time accuracy. The higher order nature of the scheme allows the numerical coupling between flux and source terms appearing in the governing equations and, importantly, to handle moderately stiff and stiff source terms. Stiff source terms arise in the case of abrupt changes of river geometry such as in the case of hydraulic structures like bridges and weirs. Hydraulic interpretation of these conditions with 1D numerical modelling requires particular attention; for instance, a 1st order scheme might either lead to inaccurate solutions or impossibility to simulate these complex conditions. Validation is carried out with several test cases with the aim to check the scheme capability to deal with abrupt geometric changes and to capture the direction and celerity of propagation of bed and water surface disturbances. Validation is done also in a real case by using stage-discharge field measurements in the Ombrone river (Tuscany). The proposed scheme is further employed for the computation of flow rating curves in cross-sections just upstream of an abrupt narrowing, considering both fixed and movable bed conditions and different ratios of contraction for cross-section width. This problem is of particular relevance as, in common engineering practice, rating curves are derived from stage-measuring gauges installed on bridges with flow conditions that are likely to be influenced by local width narrowing. Results show that a higher order scheme is needed in order to deal with stiff source terms and reproduce realistic flow rating curves, unless a strong refinement of the computational grid is performed. This capability appears to be crucial for the computation of rating curves on coarse grids as it allows the modeling of abrupt contractions and jumps in bed bottom elevations, which often occur near cross-sections where stage measuring gauges are installed.
\end{abstract}

(c) 2015 Elsevier Ltd. All rights reserved.

\section{Introduction}

In the present work, a higher order 1D Discontinuous Galerkin numerical scheme for the propagation of flood hydrograph over a compound channel with movable bed is presented. The scheme is designed in order to model the complex geometry variations occurring in natural channels, which are represented by source terms in the governing equations.

The model equations are represented by the coupled system of balance laws (SBL) formed by the 1D Shallow Water equations and the Exner sediments continuity equation, written for a compound

\footnotetext{
* Corresponding author. Tel.: +3955 4796458.

E-mail addresses: Iminatti@dicea.unifi.it (L. Minatti), pinandc@dicea.unifi.it (P.N. De Cicco), luca.solari@unifi.it (L. Solari).
}

natural channel. A bedload transport equation is used as closure condition for sediments dynamics.

Source terms appearing in the shallow water equations for natural channels are related to channel width, slope and friction. The presence of abrupt geometry variations, which, for instance, is common in cross-sections near stage measuring gauges in rivers, may lead to stiff source terms. The characteristic speed associated to such terms is much larger than the one associated to the flux term. If an explicit numerical scheme does not feature some appropriate treatment for stiff source terms, the numerical solution can be inaccurate and asymptotically inconsistent (see Dumbser et al. [3]) with wrong advection speed estimates or even instabilities on coarse grids.

In order to solve an SBL, a widely used method is the so-called source term splitting (see Toro [2]). It consists in splitting the SBL onto two sub-problems to be solved in sequence: a homogeneous problem, in which the source term in the original SBL is not taken into 
account, and an ODE containing only the source term and the time derivatives of state from the original SBL. The numerical method to solve the ODE can feature some appropriate treatment for stiff source terms if needed. This method is however not optimal when a scheme which is also capable of effectively reproducing stationary solutions is sought, as splitting methods may lead to oscillations near steady states.

The main reason that motivated the present work is the one of devising a scheme capable of treating moderately stiff and stiff source terms, while preserving other desired properties such as the wellbalanced property, which allows steady states to be reproduced with a certain accuracy.

The scheme has been developed within the framework of the ADER-Discontinuous Galerkin (DG) methods, as proposed by Dumbser et al. [1]. As it will be clear from Section 3, it is necessary to employ a higher order scheme (a scheme with order of accuracy higher than the 1st), in order to preserve the coupling between the flux and the source term. Also, the nature of the model equations requires the use of a path-conservative formalism in order to treat liquid and solid dynamics in a coupled way.

In the literature, 1D shallow water numerical models have been recently proposed both using 1 st order (e.g. Catella et al. [4], Audusse et al. [5]) and higher order (e.g. Caleffi et al. [6], Siviglia et al. [7] and Canestrelli et al. [8]) schemes.

It would still be possible to use a shallow water scheme not featuring a special treatment for source terms in order to simulate abrupt geometry changes in natural rivers, by refining the computational grid. However, the use of an ADER-DG strategy in such cases proves to be effective with no need for grid refinement and the resulting scheme is very stable and can be easily extended to even higher orders of accuracy.

The use of an ADER-DG strategy in the case of shallow water equations for natural channels has never been investigated in the scientific literature, to the knowledge of the authors.

The proposed numerical scheme can in principle reach any order of space-time accuracy. For the applications in the present work, the 2nd order accuracy has been found sufficient and represents a good compromise between accuracy and calculation time. This is partly due to information on river geometry being often low in accuracy.

The model is validated against several benchmarks: (i) water at rest in a non-prismatic channel, (ii) dam break problem with a moving strong shock, (iii) steady flow in a Venturi-type flume, (iv) subcritical flow in an irregular channel, and (v) propagation of a sediment hump near critical conditions. Moreover, the model is further validated against field measurements of water level-flow discharge during a flood in the Ombrone Pistoiese river in Tuscany (Italy).

The implications of modelling moderately stiff or stiff source terms in the case of flow rating curves are discussed. Importantly, conditions of non-uniformity are found in cross-sections just upstream of bridges where, typically, water-level gauges are installed for flow monitoring by making use of stage-discharge rating curves. Abrupt geometric changes, due to a rapid cross-section narrowing, can deeply affect the flow, leading for instance to backwater effects and transition to a supercritical state. As a result, rating curves may considerably deviate from the classical power law function assuming non-trivial shapes. Moreover, flow unsteadiness can produce a hysteretic behavior (see Schmidt and Yen [9] and Francalanci et al. [10]). In these conditions, reliable rating curves need to be developed by coupling filed measurements with 1D hydraulic numerical modelling.

The model is applied to the case of abrupt geometry changes, where flow can be more effectively reproduced by using a higher order scheme featuring some kind of treatment for stiff source terms; a 1 st order scheme would lead to erroneous results, unless a drastic grid refinement was performed. Flow rating curves are derived in a schematic channel subject to a flood wave forcing in the cases of movable and fixed bed with a local width constriction (such as in the case of bridge piers). Various numerical tests are carried out considering different degrees of channel narrowing for given boundary conditions (i.e. input hydrograph, upstream bed level and downstream water level). Results are shown considering the present higher order scheme and its 1st order version. It appears that in these geometrical conditions, rating curves are better reproduced only when a higher order numerical scheme is considered. Importantly, a 1st order scheme can lead to wrong/inaccurate results although it does not become unstable.

The numerical scheme is introduced in Section 3, and its validation and application to the computation of rating curves are shown in Section 4. A discussion of the results and the conclusions are shown in Section 5.

\section{Model equations}

The model features the shallow water equations for a natural channel coupled with the Exner equation describing sediments mass conservation. A capacitive approach is used in the present work: the solid flow rate in the channel is assumed to be coincident with the solid flow rate as predicted by bedload transport formulas.

The three governing equations can be written as an SBL having the following form:

$\frac{\partial U}{\partial t}+\frac{d F}{d x}=B \frac{\partial U}{\partial x}+S \frac{\partial \omega}{\partial x}$

where $(x, t)$ are the space-time coordinates, $U(x, t)$ is the state vector, $\omega(x)$ is a known scalar function useful for describing the geometry of the system, $F(U, \omega)$ is a flux vector, $B(U, \omega)$ is a coupling matrix, $S(U$, $\omega$ ) is the source term vector, and $d \cdot / d x$ indicates the total derivative operator. The total derivative is used to take into account fluxes depending on the space coordinate $x$ independently of the state $U$, as it happens in the case of channels with space varying cross-section shape.

In order to do so, the above mentioned terms are defined:

$U(x, t)=\left(\begin{array}{c}\Omega \\ Q \\ \Omega_{s}\end{array}\right)$

$\omega(x, t)=x$

$F(\omega, U)=\left(\begin{array}{c}Q \\ \beta \frac{Q^{2}}{\Omega}+g I_{0} \\ \frac{Q_{s}}{\phi}\end{array}\right)$

$B(\omega, U)=\left(\begin{array}{ccc}0 & 0 & 0 \\ 0 & 0 & -\frac{g \Omega}{L_{0}} \\ 0 & 0 & 0\end{array}\right)$

$S(\omega, U)=\left(\begin{array}{c}0 \\ g I_{1}-g \Omega \frac{\partial b_{0}}{\partial x}+g \frac{\Omega}{L_{0}}\left(b-b_{0}\right) \frac{\partial L_{0}}{\partial x}-g \Omega \frac{Q^{2}}{K_{c}^{2}} \\ 0\end{array}\right)$

where $\Omega$ is the wetted cross-section area, $Q$ is the liquid discharge, $\Omega_{s}$ is the solid area that is scoured or aggraded by the flow, $Q_{S}$ is the (volumetric) solid flow rate, $\phi$ is the volume fraction of the bed material (defined as the volume occupied by the solid grains on the unit bulk volume of bed material), $\beta$ is the Boussinesq coefficient accounting for non uniform velocity distribution on the cross-section, $K_{c}$ is the cross-section conveyance, $g$ is the gravity acceleration, $b$ is the channel thalweg elevation. 


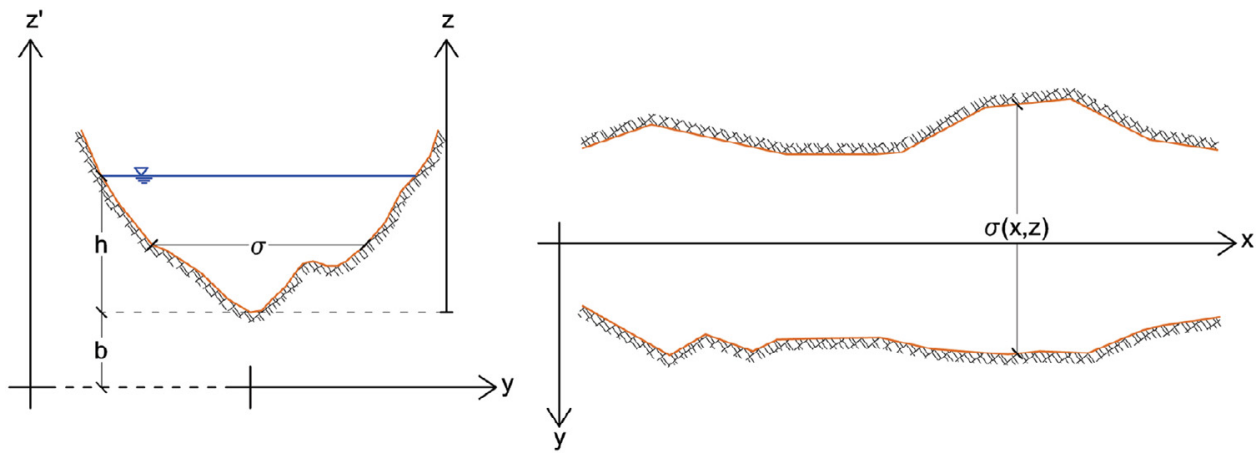

Fig. 1. Definition sketch for the shallow water equations. A $x=$ const. plane is shown on the left, while a $z=$ const. plane is shown on the right.

The volume fraction $\phi$ is assumed to be a constant (it is usually assumed to be around 0.6 ) and is taken into the flux vector.

The $\beta$ and $K_{c}$ terms are calculated according to the method employed for compound channel modelling. The well known Divided Channel Method (DCM) by Chow [11] is used here, leading to the following expressions for such coefficients:

$K_{c}=\sum_{i} \frac{\Omega_{i}^{5 / 3}}{n_{i} \cdot P_{i}^{2 / 3}} \quad i=l o b$, chn, rob

where $\Omega_{i}, P_{i}$ and $n_{i}$ are respectively the wetted area, the wetted perimeter and the Manning coefficient in either the left overbank (lob), the main channel (chn) or the right overbank (rob) compartment of the cross-section.

$\beta=\frac{\Omega}{K_{c}^{2}} \cdot \sum_{i} \frac{\Omega_{i}^{7 / 3}}{n_{i}^{2} P_{i}^{4 / 3}}$

The $L_{0}$ and the $b_{0}$ terms represent, respectively, the channel top width and thalweg elevation at a time $t_{0}<t$, with $t$ being the current simulation time. Such quantities are used to represent the erosional/aggradational history of a cross-section prior to time $t$. In practice, $t_{0}$ is assumed equal to time $t^{n-1}$ in the explicit time stepping scheme, $t^{n}$ being the current time step.

Finally, the $I_{0}$ and $I_{1}$ terms are calculated as follows:

$I_{0}=\int_{0}^{h}(h-z) \sigma d z$

$I_{1}=\int_{0}^{h}(h-z) \frac{\partial \sigma}{\partial x} d z$

where $h$ is the cross-section hydraulic depth, $z$ is the coordinate along a vertical axis whose origin is placed at the bottom of cross-section (local height), $\sigma$ is the local cross-section breadth in $(x, z)$.

Fig. 1 shows the definition sketch for the problem:

Note that it is not possible to write the $\left(g \Omega / L_{0}\right) \cdot\left(\partial \Omega_{s} / \partial x\right)$ term appearing into the $B \cdot(\partial U / \partial x)$ product of Eq. (1) as the $x$-derivative of some quantity: the model equations are a nonconservative SBL and they can not be written without using a coupling matrix $B$.

The quasi-linear form of the model equations is found by observing that the flux total derivative can be written as:

$\frac{d F}{d x}=J \frac{\partial U}{\partial x}+V \frac{\partial \omega}{\partial x}$

where $J=\partial F / \partial U$ is the flux Jacobian matrix and $V=\partial F / \partial \omega$ is the geometry variation term.

In the proposed model, river overbanks are considered to act as fixed bed portions of the river cross-section, while the main channel is left free to modify its morphology according to the hydraulic conditions.
In order to restrict the river morphological changes to the main channel area, bedload transport equations are calculated on the projection of hydraulic variables over the main channel. This is accomplished by using the DCM method for splitting the cross-section total flow rate into fractions relevant to overbanks and main channel compartments (the solid flow rate calculated in this way is indicated using the chn subscript). The cross-section wetted area is split onto the compartments according to the geometry of the cross-section.

The $J$ and $V$ terms have the following expressions:

$J(\omega, U)=\left(\begin{array}{ccc}0 & 1 & 0 \\ g \frac{\Omega}{\sigma_{T}}\left(1+\frac{Q^{2}}{g \Omega^{2}} \frac{\partial \beta}{\partial z}\right)-\beta \frac{Q^{2}}{\Omega^{2}} & 2 \beta \frac{Q}{\Omega} & 0 \\ \frac{1}{\phi} \frac{\partial Q_{s, c h n}}{\partial \Omega} & \frac{1}{\phi} \frac{\partial Q_{s, c h n}}{\partial Q} & 0\end{array}\right)$

$V(\omega, U)=\left(\begin{array}{c}0 \\ g I_{1}-g \frac{\Omega H_{1}}{\sigma_{T}}+\left.\frac{Q^{2}}{\Omega} \frac{\partial \beta}{\partial x}\right|_{\Omega} \\ \left.\frac{1}{\phi} \frac{\partial Q_{s, c h n}}{\partial x}\right|_{\Omega}\end{array}\right)$

where:

$H_{1}=\int_{0}^{h} \frac{\partial \sigma}{\partial x} d z$

$\sigma_{T}$ indicates the top width of the free surface and the $\left.\right|_{\Omega}$ symbol indicates differentiation at constant $\Omega$.

By substituting Eq. (11) in Eq. (1) the quasi-linear form of the SBL is obtained:

$\frac{\partial U}{\partial t}+A \frac{\partial U}{\partial x}=(S-V) \frac{\partial \omega}{\partial x}$

where:

$A=J-B$

It can be shown with some calculations that in fixed bed conditions the system is strictly hyperbolic, being the three eigenvalues of A distinct and real, except in critical conditions, where two of them coincide.

In movable bed conditions, proof of hyperbolicity in the case of Grass [12] bedload transport equation can be found in Castro et al. [13]. As far as different bedload transport formulas are concerned, an analysis by Cordier et al. [14] shows that the system is hyperbolic in most physical situations. However, in the movable bed simulations performed for the present work, only real and distinct eigenvalues were found: for $Q>0$ one of them was negative, while the remaining two were positive. 


\section{Numerical scheme}

In order to solve a hyperbolic SBL in the form of Eq. (1), the main idea is the one of writing it as an homogenous nonconservative system. The following trivial equation is therefore added to the system:

$$
\frac{\partial \omega}{\partial t}=0
$$

and an extended state vector is defined:

$\widetilde{U}=\left(\begin{array}{l}U \\ \omega\end{array}\right)$

If $U \in \mathbb{R}^{D}$, then $\widetilde{U} \in \mathbb{R}^{D+1}$.

The following homogeneous hyperbolic system of equations is considered:

$$
\frac{\partial \widetilde{U}}{\partial t}+\widetilde{A}(\widetilde{U}) \frac{\partial \widetilde{U}}{\partial x}=0
$$

If Eq. (19) represents a nonconservative system (NCS), then the $\widetilde{A}(\widetilde{U})$. $\partial \widetilde{U} / \partial x$ term cannot be written as the gradient of some flux vector and it is called a nonconservative product. Otherwise, if it represents a system of conservation laws (SCL), the term can be written as an extended flux gradient term $\partial \widetilde{F} / \partial x$.

The SBL of Eq. (1) and the trivial equation of Eq. (17) can now be written in the compact form of Eq. (19) by using the following $(D+1) \times(D+1)$ system matrix:

$\widetilde{A}=\left(\begin{array}{cc}A & -(S-V) \\ 0 & 0\end{array}\right)$

The $(S-V) \cdot(\partial \omega / \partial x)$ term acts as a nonconservative product, in addition to the $B \cdot(\partial U / \partial x)$ term, for the modified system of Eq. (20).

We use the convention of removing the sign to either indicate the top left $D \times D$ block of matrix $\widetilde{A}$ or the first $D$ components of states, without including the geometry variable $\omega$.

The numerical scheme has been built starting from the ADER-FV framework proposed by Titarev and Toro [15].

ADER-FV schemes use the solution of generalized Riemann problems at cell interfaces to achieve high-order accuracy in space and time. A difficulty in this approach is represented by the CauchyKovalewsky procedure, which is needed to obtain expressions for higher order time derivatives of states. The procedure requires successive analytical differentiations and it is rather cumbersome. In order to avoid the analytical calculations involved with it, a method based on local DG predictors was proposed by Dumbser et al. [16] for the case of a SCL with non stiff source terms and by Dumbser et al. [3] in the case of a SCL with stiff source terms. The method was then extended to nonconservative systems by Dumbser et al. [1].

In the present work, the ADER strategy involving local DG predictors is employed to devise a higher order DG scheme with moderately stiff or stiff source term treatment. While being natural to read the equations of the scheme considering its order of accuracy to be higher than the 1st, they are quite general and can be easily "contracted" to obtain a 1 st order scheme. The strategy produces a wellbalanced scheme capable of reproducing steady states.

The scheme is presented for an arbitrary order of accuracy even though its equations and applications are shown for a 2 nd order case. In the applications, the numerical solution is therefore represented by a 1 st order piecewise space polynomial at each time step.

\subsection{ADER schemes for nonconservative hyperbolic systems}

The homogeneous hyperbolic system of Eqs. (19) and (20) is considered. In order to simplify the notation we introduce the cell spacetime element as the set $E_{i}=\left[x_{i-1 / 2}, x_{i+1 / 2}\right] \times\left[t^{n}, t^{n+1}\right]$. It is convenient to normalize $E_{i}$ onto the unit space-time element $\epsilon_{i}=[0,1] \times$
$[0,1]$ through the following transformation:

$$
\begin{aligned}
\xi & =\frac{\left(x-x_{i-1 / 2}\right)}{\Delta x_{i}} \\
\tau & =\frac{\left(t-t^{n}\right)}{\Delta t}
\end{aligned}
$$

The numerical solution $\widetilde{U}_{h, i}^{n}(\xi)$ for the state $\widetilde{U}$ on the cell $\left[x_{i-1 / 2}, x_{i+1 / 2}\right]$ at time $t^{n}$ is a polynomial of order $N_{0}-1$, for a scheme with order of accuracy $N_{0}$ in space. It can therefore be expressed as a linear combination of $N_{0}$ space bases functions $\phi_{l}(\xi)$ for the set of polynomials of order $N_{0}-1$ :

$\widetilde{U}_{h, i}^{n}(\xi)=\phi_{l}(\xi) \widetilde{U}_{l, i}^{n}$

The convention of indicating sums with repeated indexes is used. The $\widetilde{U}_{l, i}^{n}=\left(U_{l, i}^{n}, \omega_{l, i}^{n}\right)$ terms in the sum are numerical coefficients used to express the numerical solution as a linear combination of the space bases.

The space bases used in the present work are orthonormal modal bases. Therefore, the first component $\widetilde{U}_{1, i}^{n}$ of the solution represents the cell average, while for instance, the second one represents its slope times the cell size $\Delta x_{i}$ and a normalization factor. Further details can be found in Appendix A.

$\widetilde{A}$ is a $(D+1) \times(D+1)$ matrix and $\widetilde{U}, \widetilde{U}_{h, i}^{n}, \widetilde{U}_{l, i}^{n}$ are $D+1$ components states.

The scheme consists of three steps: namely a reconstruction step, a predictor step, that allows to treat either moderately stiff or stiff source terms in the SBL and a fully explicit corrector step which is subject a CFL condition.

\subsection{Reconstruction step}

In order to limit the oscillations in the presence of moving shock waves, the numerical solution $\widetilde{U}_{h, i}^{n}(\xi)$ should be reconstructed in a nonlinear way by using an appropriate limiter. In the present work, the TVB limiter of Cockburn and Shu [19] and Cockburn et al. [20] has been implemented. The procedure for a 2 nd order scheme is briefly recalled.

A modified minmod function is introduced:

$m(a, b, c)= \begin{cases}a & \text { if }|a|<K \cdot \Delta X^{2} \\ \operatorname{minmod}(a, b, c) & \text { else }\end{cases}$

where $K$ is a problem dependent parameter used to preserve local extremes, which should be set according to the maximum value of the second derivative of initial conditions near critical points (see Cockburn and Shu [19]), and $\Delta X$ is the maximum grid spacing.

The following modified interface values are computed for the solution in the cell:

$$
\begin{aligned}
& r_{i+1 / 2}^{-}=\widetilde{U}_{1, i}^{n}+m\left(\widetilde{U}_{h, i}^{n}\left(1^{-}\right)-\widetilde{U}_{1, i}^{n}, \widetilde{U}_{1, i}^{n}-\widetilde{U}_{1, i-1}^{n}, \widetilde{U}_{1, i+1}^{n}-\widetilde{U}_{1, i}^{n}\right) \\
& r_{i-1 / 2}^{+}=\widetilde{U}_{1, i}^{n}+m\left(\widetilde{U}_{h, i}^{n}\left(0^{+}\right)-\widetilde{U}_{1, i}^{n}, \widetilde{U}_{1, i}^{n}-\widetilde{U}_{1, i-1}^{n}, \widetilde{U}_{1, i+1}^{n}-\widetilde{U}_{1, i}^{n}\right)
\end{aligned}
$$

If both $r_{i+1 / 2}^{-}=\widetilde{U}_{h, i}^{n}\left(1^{-}\right)$and $r_{i-1 / 2}^{+}=\widetilde{U}_{h, i}^{n}\left(0^{+}\right)$, no reconstruction is performed. Otherwise, the following reconstructed slope is computed for the solution:

$s_{i}^{R}=m\left(\frac{\widetilde{U}_{h, i}^{n}\left(1^{-}\right)-\widetilde{U}_{h, i}^{n}\left(0^{+}\right)}{\Delta x_{i}}, \frac{\widetilde{U}_{1, i}^{n}-\widetilde{U}_{1, i-1}^{n}}{\Delta x_{i}+\Delta x_{i-1}}, \frac{\widetilde{U}_{1, i+1}^{n}-\widetilde{U}_{1, i}^{n}}{\Delta x_{i+1}+\Delta x_{i}}\right)$

and the initial solution $\widetilde{U}_{h, i}^{n}(\xi)$ is replaced by the following:

$\widetilde{U}_{h, i}^{R, n}(\xi)=\widetilde{U}_{1, i}^{n}+s_{i}^{R} \cdot \Delta x_{i} \cdot\left(\xi-\frac{1}{2}\right)$

The $\widetilde{U}_{2, i}$ component of the reconstructed solution with respect to the modal bases can then be calculated from the $\mathcal{L}_{2}$ projection of $\widetilde{U}_{h, i}^{R, n}$. 
For schemes of order higher than the 2nd, the above procedure is applied to the $\mathcal{L}_{2}$ projection of $\widetilde{U}_{h, i}^{n}(\xi)$ onto the space of polynomials of degree one. For 1st order schemes, the whole procedure can't be applied and it is therefore skipped.

If the treatment of strong moving shocks is not needed, the present step can be skipped and the solution $\widetilde{U}_{h, i}^{n}(\xi)$ can be directly used into the predictor step of Section 3.3. The scheme, when used with no limiter, is a linear DG scheme: strong moving shocks can't be reproduced even though it is known from the literature, see Atkins and Shu [21], that linear DG schemes still perform rather well in the presence of non strong discontinuities.

In order to simplify the notation, both the reconstructed (DG scheme with TVB limiter) and the non reconstructed solutions (linear DG scheme) will be indicated with $\widetilde{U}_{h, i}^{n}(\xi)$ throughout the rest of the paper.

\subsection{Predictor step}

The Cauchy-Kovalewsky procedure is needed in ADER-FV schemes to achieve higher order accuracy in time. It is here replaced by a purely numerical procedure based on a local weak formulation of the PDE in the space-time element.

An unknown state $\widetilde{W}$, whose numerical approximation is indicated with $\widetilde{W}_{h, i}(\xi, \tau)=\left(W_{h, i}, \mu_{h, i}\right)$, is considered in the space-time element $\epsilon_{i} \cdot \widetilde{W}_{h, i} \neq \widetilde{U}_{h, i}$ in general.

For a model with order of accuracy $N-1$ in both space and time, $\widetilde{W}_{h, i}(\xi, \tau)$ is a polynomial of order $N-2$ in $(\xi, \tau) . \widetilde{W}_{h, i}(\xi, \tau)$ is written as a linear combination of $N$ space-time bases $\theta(\xi, \tau)$ which are space-time polynomials of order $N-2$. The following notation is used:

$\widetilde{W}_{h, i}(\xi, \tau)=\theta_{l}(\xi, \tau) \cdot \tilde{q}_{l, i}$

The $\widetilde{q}_{l, i}=\left(q_{l, i}, \mu_{l, i}\right)$ terms in the sum are unknown numerical coefficients used to express the numerical solution as a linear combination of the space-time bases. Both $\widetilde{W}_{h, i}$ and $\widetilde{q}_{l, i}$ are $D+1$ components states.

Note that $N>N_{0}$ in general. Also, for a 2nd order scheme $N_{0}=2$ and $N=3$.

The space-time bases used in the present work are orthonormal modal bases. Further details can be found in Appendix A.

Predictor step is performed by multiplying Eq. (19) for a test function and by integrating on the internal part of $\epsilon_{i}$ to obtain a weak formulation of the PDE. Integration is performed on the internal part of the element, which we indicate with $\dot{\epsilon}_{i}=(0,1) \times(0,1)$ as the predictor is a local operator and coupling with neighboring cells is not considered at this stage. We use the space-time bases functions $\theta(\xi$, $\tau$ ) as test functions and substitute the exact state $\widetilde{W}$ with its numerical approximation $\widetilde{W}_{h, i}$. The following weak formulation is obtained:

$\iint_{\dot{\epsilon}_{i}} \theta_{k} \frac{\partial \widetilde{W}_{h, i}}{\partial \tau} d \xi d \tau+\iint_{\dot{\epsilon}_{i}} \theta_{k} \widetilde{A}^{*} \frac{\partial \widetilde{W}_{h, i}}{\partial \xi} d \xi d \tau=0$

where $\widetilde{A}^{*}=\tilde{A} \Delta t / \Delta x_{i}$.

The 2nd term of Eq. (29) allows the coupling between the flux and the source terms. It can be observed how this is possible only for a higher order scheme as it would be $\partial \widetilde{W}_{h, i} / \partial \xi=0$ in the case of a 1 st order scheme and Eq. (29) would reduce to a trivial equation.

The value of $\Delta t$ is obtained at the beginning of the predictor step from a CFL condition based on the eigenvalues of matrix $A$, calculated on the numerical solution $\widetilde{U}_{h}^{n}$.

The $\widetilde{A}^{*} \cdot\left(\partial \widetilde{W}_{h, i} / \partial \xi\right)$ term is projected onto the space of space-time polynomials of order $N-2$ on the unit space-time element using the $L_{2}$ projection with modal bases. This provides an approximation of the term as $\widetilde{A}^{*}$ is in general a nonlinear function of $\widetilde{W}_{h, i}$. It is then possible to express the projected term as a linear combination of the space-time modal bases:

$\tilde{A}^{*} \frac{\partial \tilde{W}_{h, i}}{\partial \xi}=\tilde{p}_{l, i} \cdot \theta_{l}$

The expressions for the $\widetilde{p}_{l, i}$ coefficients can be found by evaluating the inner product of the term with each modal basis:

$\widetilde{p}_{k, i}=\iint_{\dot{\epsilon}_{i}} \tilde{A}^{*} \frac{\partial \widetilde{W}_{h, i}}{\partial \xi} \cdot \theta_{k} d \xi d \tau$

In general, the $\tilde{p}_{k, i}$ coefficients are nonlinear functions of all the $\tilde{q}_{l, i}, l=1 . . N$ coefficients.

The integral in Eq. (31) can be numerically approximated via Gaussian quadrature. The number of quadrature points should be chosen according to the accuracy of the scheme, bearing in mind that with $N_{G}$ Gaussian points, integration is exact for polynomials up to order $2 N_{G}-1$. For a 2 nd order accurate scheme and for a linear hyperbolic system, a single Gaussian quadrature point located in $(\xi, \tau)=$ $(1 / 2,1 / 2)$ usually allows for a sufficiently accurate integration (even if the integrand in Eq. (31) would be a quadratic space-time function). In the case of a nonlinear system such as the one under study, the coefficients inside the $\widetilde{A}^{*}$ matrix are nonlinear functions of the state components. In this case, the number of Gaussian points to be used should depend on how high the nonlinearity of the coefficients is. For geometries with simple cross-sections (e.g. rectangular) we found the use of one Gaussian point to provide sufficient accuracy. For more complex geometries, such as the ones in natural channels, the use of more Gaussian points should be considered at the price of a more time consuming scheme.

If the $(D+1)$ th component of Eq. (29) is considered, then the following is obtained:

$\iint_{\dot{\epsilon}_{i}} \theta_{k} \frac{\partial \mu_{h, i}}{\partial \tau} d \xi d \tau=0$

As Eq. (32) is verified for each space-time basis $\theta_{k}$, it is concluded that the $(D+1)$ th component $\mu_{h}$ of the predicted state $\widetilde{W}_{h}$ does not depend on the normalized time $\tau$. This is an expected result as Eq. (29) is the local weak formulation of Eq. (19) whose $(D+1)$ th row reads as $\partial \omega / \partial t=0$.

The step proceeds in two different ways, according to the stiffness of the source/geometry variation term $S-V$.

\subsubsection{Non stiff or moderately stiff source term}

The expressions of Eqs. (28) and (30) are substituted into Eq. (29) to obtain:

$K_{k l}^{\tau} \widetilde{q}_{l, i}+M_{k l} \tilde{p}_{l, i}\left(\tilde{q}_{1, i} \ldots \tilde{q}_{N, i}\right)=0$

where $K_{k l}^{\tau}$ and $M_{k l}$ are $N \times N$ matrices defined as follows:

$K_{k l}^{\tau}=\iint_{\dot{\epsilon}_{i}} \theta_{k} \frac{\partial \theta_{l}}{\partial \tau} d \xi d \tau$

$M_{k l}=\iint_{\dot{\epsilon}_{i}} \theta_{k} \theta_{l} d \xi d \tau$

$K_{k l}^{\tau}$ and $M_{k l}$ depend on the chosen space-time bases only and can be precalculated and stored in order to speed up the computations. Expressions for such matrices for $N=3$ (2nd order scheme) are shown in Appendix A.

Eq. (33) is a nonlinear algebraic equation. The unknowns are represented by the $\widetilde{q}_{l, i}$ coefficients. In this case, the $\widetilde{q}_{l, i}$ components relevant to pure spatial modes are obtained from the initial condition:

$\widetilde{W}_{h, i}(\xi, 0)=\tilde{U}_{h, i}^{n}(\xi)$

The remaining $\widetilde{q}_{l, i}$ components are obtained by directly solving Eq. (33).

The expression of Eq. (33) in the case of a 2nd order scheme can be found in Appendix B for the model equations under study. 


\subsubsection{Stiff source terms}

The first term of Eq. (29) is integrated by parts in time. The initial state $\widetilde{U}_{h, i}^{n}(\xi)$ is used to define the initial condition into the term integrated by parts:

$$
\begin{aligned}
& \int_{0}^{1} \theta_{k}(\xi, 1) \widetilde{W}_{h, i}(\xi, 1) d \tau-\int_{0}^{1} \theta_{k}(\xi, 0) \widetilde{U}_{h, i}(\xi) d \tau \\
& -\iint_{\dot{\epsilon}_{i}} \frac{\partial \theta_{k}}{\partial \tau} \widetilde{W}_{h, i} d \xi d \tau+\iint_{\dot{\epsilon}_{i}} \theta_{k} \widetilde{A}^{*} \frac{\partial \widetilde{W}_{h, i}}{\partial \xi} d \xi d \tau=0
\end{aligned}
$$

Performing integration by parts in time implies that the predicted state $\widetilde{W}_{h, i}$ may exhibit a discontinuity in time at $\tau=0$, that is $\widetilde{W}_{h, i}(\xi, 0) \neq \widetilde{U}_{h, i}^{n}(\xi)$. The discontinuity is induced by the presence of the stiff source terms locally acting in the element.

By substituting the expression of Eq. (28) for $\widetilde{W}_{h, i}$, the expression of Eq. (22) for $\widetilde{U}_{h, i}$ and Eq. (30) in Eq. (37), one obtains:

$M_{k l}^{(1)} \widetilde{q}_{l, i}-M_{k l}^{(0)} \widetilde{U}_{l, i}^{n}-{ }^{t}\left(K_{k l}^{\tau}\right) \widetilde{q}_{l, i}+M_{k l} \tilde{p}_{l, i}\left(\widetilde{q}_{1, i} \ldots \widetilde{q}_{N, i}\right)=0$

where the superscript ' $t$ ' indicates the transpose operator and $M_{k l}^{(1)}$ and $M_{k l}^{(0)}$ are respectively $N \times N$ and $N \times N_{0}$ matrices, defined as follows:

$M_{k l}^{(1)}=\int_{0}^{1} \theta_{k}(\xi, 1) \theta_{l}(\xi, 1) d \xi$

$M_{k l}^{(0)}=\int_{0}^{1} \theta_{k}(\xi, 0) \phi_{l}(\xi) d \xi$

$M_{k l}^{(1)}$ and $M_{k l}^{(0)}$ depend on the chosen bases only and can be precalculated and stored. Their expression is shown in Appendix A for $N=3$ (2nd order scheme).

As opposite to the non stiff source term case, all of the $\widetilde{q}_{l, i}$ components are obtained from the solution of Eq. (38), while the initial condition is taken into account by the 2nd term on the LHS of the equation. A time discontinuity at $\tau=0$ is admitted in this case and thus $\widetilde{q}_{l, i} \neq \widetilde{U}_{l, i}^{n}, \quad l=1, N_{0}$ in general.

The expression of Eq. (38) in the case of a 2nd order scheme can be found in Appendix B for the model equations under study.

\subsection{Corrector step}

The final step of the scheme, that is the corrector step, is an explicit time step evolving the numerical solution $\widetilde{U}_{h, i}^{n}$ onto time step $t^{n+1}$.

Eq. (19) is multiplied by the space bases test functions $\phi(\xi)$ and integrated on $E_{i}$. At this stage, the boundaries of the space-time element are involved in the integration process in order to consider the coupling with the neighboring cells. The numerical approximation $\widetilde{U}_{h, i}$ of $\widetilde{W}$ in cell $i$ is used in the term involving the time derivative, while the numerical approximation $\widetilde{W}_{h, i}$ obtained from the predictor step is used in the term involving the space derivative. Integration by parts in time (bear in mind that the space bases functions $\phi_{k}$ depend on $\xi$ only) is also performed. The following equation is obtained:

$\int_{0}^{1} \phi_{k} \widetilde{U}_{h, i}^{n+1} d \xi-\int_{0}^{1} \phi_{k} \widetilde{U}_{h, i}^{n} d \xi+\frac{\Delta t}{\Delta x_{i}} \iint_{\epsilon_{i}} \phi_{k} \widetilde{A} \frac{\partial \widetilde{W}_{h, i}}{\partial \xi} d \xi d \tau=0$

The integral term involving the space derivatives in the equation can be split into two parts: a local part, where integration is performed in $\dot{\epsilon}_{i}$ and in a boundary part $\partial \dot{\epsilon}_{i}$ providing the coupling with the neighboring cells.

The boundary part accounts for jumps in the solution at the boundaries with neighboring cells and is here treated with a pathconservative Roe-scheme [22]. A path connecting states at opposite sides of interfaces is therefore chosen in order to account for nonconservative products and Riemann solvers are introduced.
As far as the integration path is concerned, a linear path is chosen:

$$
\begin{aligned}
& \widetilde{\Psi}_{i+1 / 2}\left(s, \widetilde{W}_{h, i}\left(1^{-}, \tau\right), \widetilde{W}_{h, i+1}\left(0^{+}, \tau\right)\right)=\widetilde{W}_{h, i}\left(1^{-}, \tau\right) \\
& \quad+s \cdot\left(\widetilde{W}_{h, i+1}\left(0^{+}, \tau\right)-\widetilde{W}_{h, i}\left(1^{-}, \tau\right)\right)
\end{aligned}
$$

The choice of an integration path, such as the one of Eq. (42) allows the definition of a generalized Roe matrix:

$\tilde{A}_{i+1 / 2}(\tau)=\int_{0}^{1} \tilde{A}\left(\tilde{\Psi}_{i+1 / 2}\left(s, \widetilde{W}_{h, i}^{n}\left(1^{-}, \tau\right), \tilde{W}_{h, i+1}^{n}\left(0^{+}, \tau\right)\right)\right) d s$

A Riemann solver provides an approximate solution of the Riemann problems arising at cell interfaces thus allowing to calculate the upwinding matrices $\widetilde{A}_{i+1 / 2}^{+}$and $\widetilde{A}_{i+1 / 2}^{-}$at each cell interface. The upwinding matrices allow to integrate the equations according to the direction towards which information are propagated by the system.

One of the most common Riemann solver is the solver of Roe [23]. It is a linear solver with low numerical diffusivity. Being a linear solver, it has the drawback of computing entropy-violating shocks: the issue can however be fixed by using an entropy fix, such as the one proposed by Harten and Hyman [24].

The expression for the Roe solver upwinding matrices for systems like the one of Eq. (20) has been devised by Pares and Castro [22] and is shown below for completeness:

$\tilde{A}_{i+1 / 2}^{ \pm}(\tau)=\left(\begin{array}{cc}\frac{A_{i+1 / 2} \pm|A|_{i+1 / 2}}{2} & -P_{i+1 / 2}^{ \pm} \cdot\left(S_{i+1 / 2}-V_{i+1 / 2}\right) \\ 0 & 0\end{array}\right)$

where $\left|A_{i+1 / 2}\right|=R_{i+1 / 2} \cdot\left|\Lambda_{i+1 / 2}\right| \cdot R_{i+1 / 2}^{-1}$ with $R_{i+1 / 2}$ being the matrix whose columns are the eigenvectors of $A_{i+1 / 2}$ and $\Lambda_{i+1 / 2}$ being the diagonal matrix with the eigenvalues $\lambda_{k}$ of $A_{i+1 / 2}$ on the diagonal. The $P_{i+1 / 2}^{ \pm}$matrices are called projection matrices and are calculated as follows:

$P_{i+1 / 2}^{ \pm}(\tau)=\frac{I \pm R_{i+1 / 2} \cdot \operatorname{sgn}\left(\Lambda_{i+1 / 2}\right) \cdot R_{i+1 / 2}^{-1}}{2}$

$\operatorname{sgn}\left(\Lambda_{i+1 / 2}\right)$ is a diagonal matrix such that $\operatorname{sgn}\left(\Lambda_{i+1 / 2}\right)_{k k}=\left|\lambda_{k}\right| / \lambda_{k}$ if $\lambda_{k} \neq 0$ and $\operatorname{sgn}\left(\Lambda_{i+1 / 2}\right)_{k k}=0$ otherwise. $I$ is the unit matrix. Furthermore:

$S_{i+1 / 2}(\tau)=\int_{0}^{1} S\left(\tilde{\Psi}\left(s, \widetilde{W}_{h, i}^{n}\left(1^{-}, \tau\right), \widetilde{W}_{h, i+1}^{n}\left(0^{+}, \tau\right)\right)\right) d s$

$V_{i+1 / 2}(\tau)=\int_{0}^{1} V\left(\tilde{\Psi}\left(s, \widetilde{W}_{h, i}^{n}\left(1^{-}, \tau\right), \tilde{W}_{h, i+1}^{n}\left(0^{+}, \tau\right)\right)\right) d s$

Another widely used Riemann solver is the Osher solver, firstly proposed by Engquist and Osher [25]. The solver is nonlinear and as opposite to the Roe solver, does not have the drawback of computing entropy-violating shocks. Dumbser and Toro [26] proposed a pathconservative version of the solver for a nonconservative homogenous system and showed its application to the case of the shallow water equations for a prismatic channel with a rectangular cross-section.

The Osher upwinding matrix for a system like the one of Eq. (20) is therefore calculated from the following:

$\widetilde{A}_{i+1 / 2}^{ \pm}=\widetilde{A}_{i+1 / 2} \pm \int_{0}^{1}\left|\widetilde{A}\left(\widetilde{\Psi}\left(s, \widetilde{U}_{i}^{n}, \widetilde{U}_{i+1}^{n}\right)\right)\right| d s$

and can now be used to implement the Osher solver in the case of shallow water equations for a non prismatic (natural) channel.

After some straightforward calculations starting from Eq. (20), the upwinding matrices for the Osher solver turn out to be 
as follows:

$$
\begin{aligned}
\tilde{A}_{i+1 / 2}^{ \pm}(\tau)= & \int_{0}^{1}\left(\begin{array}{cc}
\frac{A \pm|A|}{2}-P^{ \pm} \cdot(S-V) & \\
0 & 0
\end{array}\right) \\
& \left(\tilde{\Psi}\left(s, \tilde{W}_{h, i}^{n}\left(1^{-}, \tau\right), \tilde{W}_{h, i+1}^{n}\left(0^{+}, \tau\right)\right)\right) d s
\end{aligned}
$$

In order to make the notation independent from the used Riemann solver, the upwinding matrices are written in the following way for each solver:

$\tilde{A}_{i+1 / 2}^{ \pm}(\tau)=\left(\begin{array}{cc}A_{i+1 / 2}^{ \pm} & -G_{i+1 / 2}^{ \pm} \\ 0 & 0\end{array}\right)$

For the Roe solver, the $A_{i+1 / 2}^{ \pm}$matrix is calculated as in the upper left $D \times D$ block in the RHS of Eq. (44) and the $G_{i+1 / 2}^{ \pm}$vector is calculated as follows:

$G_{i+1 / 2}^{ \pm}(\tau)=P_{i+1 / 2}^{ \pm} \cdot\left(S_{i+1 / 2}-V_{i+1 / 2}\right)$

For the Osher solver, the $A_{i+1 / 2}^{ \pm}$matrix is calculated as in the upper left $D \times D$ block in the RHS of Eq. (49) and the $G_{i+1 / 2}^{ \pm}$vector is calculated as follows:

$G_{i+1 / 2}^{ \pm}(\tau)=\int_{0}^{1} P^{ \pm} \cdot(S-V)\left(\tilde{\Psi}\left(s, \tilde{W}_{h, i}^{n}\left(1^{-}, \tau\right), \tilde{W}_{h, i+1}^{n}\left(0^{+}, \tau\right)\right)\right) d s$

Using either Eq. (44) or Eq. (49) according to the chosen Riemann solver, Eq. (41) can be expanded:

$$
\begin{aligned}
& \int_{0}^{1} \phi_{k} \widetilde{U}_{h, i}^{n+1} d \xi-\int_{0}^{1} \phi_{k} \widetilde{U}_{h, i}^{n} d \xi+\frac{\Delta t}{\Delta x_{i}} \cdot \iint_{\dot{\epsilon}_{i}} \phi_{k} \widetilde{A} \frac{\partial \widetilde{W}_{h, i}}{\partial \xi} d \xi d \tau \\
& \frac{\Delta t}{\Delta x_{i}} \cdot \phi_{k}(0)\left(\int_{0}^{1} \widetilde{A}_{i-1 / 2}^{+}(\tau) d \tau\right) \cdot\left(\widetilde{W}_{h, i}\left(0^{+}, \tau\right)-\widetilde{W}_{h, i-1}\left(1^{-}, \tau\right)\right) \\
& \frac{\Delta t}{\Delta x_{i}} \cdot \phi_{k}(1)\left(\int_{0}^{1} \widetilde{A}_{i+1 / 2}^{-}(\tau) d \tau\right) \cdot\left(\widetilde{W}_{h, i+1}\left(0^{+}, \tau\right)-\widetilde{W}_{h, i}\left(1^{-}, \tau\right)\right)=0
\end{aligned}
$$

By substituting the expression of Eq. (22) for $\widetilde{U}_{h}$ in Eq. (53) and by using Eq. (50), one obtains the final expression for the corrector explicit time step:

$$
\begin{aligned}
& M_{k l}^{0} \cdot\left(\widetilde{U}_{l, i}^{n+1}-\widetilde{U}_{l, i}^{n}\right)+\frac{\Delta t}{\Delta x_{i}} \cdot \mathcal{L}_{k, i} \\
& \frac{\Delta t}{\Delta x_{i}} \cdot\left(\phi_{k}(0)\left(\mathcal{H}_{i-1 / 2}^{+}-\mathcal{I}_{i-1 / 2}^{+}\right)+\phi_{k}(1)\left(\mathcal{H}_{i+1 / 2}^{-}-\mathcal{I}_{i+1 / 2}^{-}\right)\right)=0
\end{aligned}
$$

where

$$
\begin{aligned}
& \mathcal{L}_{k, i}=\iint_{\dot{\epsilon}_{i}} \phi_{k} \cdot \tilde{A}\left(\tilde{W}_{h, i}\right) \cdot \frac{\partial \widetilde{W}_{h, i}}{\partial \xi} d \xi d \tau \\
& \mathcal{H}_{i+1 / 2}^{ \pm}=\int_{0}^{1} A_{i+1 / 2}^{ \pm}(\tau) \cdot\left(W_{h, i+1}\left(0^{+}, \tau\right)-W_{h, i}\left(1^{-}, \tau\right)\right) d \tau \\
& \mathcal{I}_{i+1 / 2}^{ \pm}=\left(\int_{0}^{1} G_{i+1 / 2}^{ \pm}(\tau) d \tau\right) \cdot\left(\mu_{h, i+1}\left(0^{+}\right)-\mu_{h, i}\left(1^{-}\right)\right)
\end{aligned}
$$

Eq. (54) provides an explicit scheme which is stable under a CFL condition. The theoretical Courant number limits are $C F L<0.32$ for a 2nd order scheme and, $C F L<0.17$ for a 3rd order scheme, according to a linear stability analysis performed by Dumbser et al. [16]. Such limits are in accordance with the usual ones for a DG scheme, depending on the degree of the polynomial representing the numerical solution. They are however lower than the ones typically needed in pure FV schemes where the numerical solution is a polynomial reconstructed starting from cell averages. On the other hand, a DG scheme is very stable, satisfying an entropy inequality on the cell (see Jiang and Shu [27]) and the whole predictor step of Section 3.3, accounting for a fair share of the computational time within the timestep can be easily arranged for a parallel code, needing only information on the local cell.

The integrals in Eqs. (55)-(57) can be numerically approximated via Gaussian quadrature. As far as the number of Gaussian quadrature points to be used is concerned, similar considerations to the ones in Section 3.3 apply.

The expression of Eq. (54) in the case of a 2nd order scheme can be found in Appendix B.

\section{Applications}

\subsection{Validation of the numerical model}

Validation of the present model is carried-out by performing numerical simulations of (i) water at rest in a non-prismatic channel, (ii) dam break problem with a moving strong shock, (iii) steady flow in a Venturi-type flume, (iv) subcritical flow in an irregular channel, (v) propagation of a sediment hump near critical conditions, and a real flood wave in the Ombrone Pistoiese river in Tuscany (Italy).

\subsubsection{Water at rest in a non-prismatic channel}

The water at rest test case of Garcia-Navarro and Vazquez-Cendon [28] is reproduced. Both the width of the rectangular cross-section of the channel (see Fig. 2) and its thalweg elevation (see Fig. 3) show abrupt linear variations in space.

The water surface elevation for the simulation is constant and set to $12 \mathrm{~m}$. The grid spacing was set to $\Delta x=5 \mathrm{~m}$ and the Roe Riemann solver was used. The results of the simulation are shown in Fig. 3.

The comparison with the analytical solution shows that the water surface is captured with very low errors by the scheme. In this test case, the wetted area $\Omega$ is a second degree polynomial in space, as both the rectangular cross-section width and the hydraulic depth are linear functions. A 3rd order scheme, which employs 2nd order polynomials to reproduce states, would be able to exactly reproduce the water at rest state, as the source and the flux terms would be exactly balanced. With a 2 nd order scheme, the errors are however bounded and grid spacing dependent, due to the well-balanced property.

\subsubsection{Dam break problem}

This test case is a dam break (Riemann) problem involving a moving shock and a rarefaction. Its purpose is the one of testing the scheme capabilities at handling moving strong shocks.

The geometry of this problem is the one of a $25 \mathrm{~m}$ long frictionless rectangular channel with unit width and flat bottom. We performed two simulations starting from the following initial conditions:

$$
(h, Q)=\left\{\begin{array}{l}
\left.\left(1.0 \mathrm{~m}, 1.25 \mathrm{~m}^{3} / \mathrm{s}\right) \text { if } x<10 \mathrm{~m} \text { (Test } A\right) \\
\left(1.0 \mathrm{~m}, 5.00 \mathrm{~m}^{3} / \mathrm{s}\right) \text { if } x<10 \mathrm{~m}(\text { Test } B) \\
\left(0.2 \mathrm{~m}, 0.00 \mathrm{~m}^{3} / \mathrm{s}\right) \text { if } x>10 \mathrm{~m}
\end{array}\right.
$$

and used free outflow boundary conditions at the ends of the channel.

The scheme with the Osher Riemann solver was used both with and without the TVB limiter of Section 3.2. The grid spacing was set to $\Delta x=0.5 \mathrm{~m}$. The results are shown in Fig. 4 . The Froude number ahead of the shock is around 1.1 for test $A$ and around 1.4 for test $B$. In the case of a milder shock (test A) both the linear DG scheme and the DG scheme with TVB limiter can reproduce the analytical solution. 


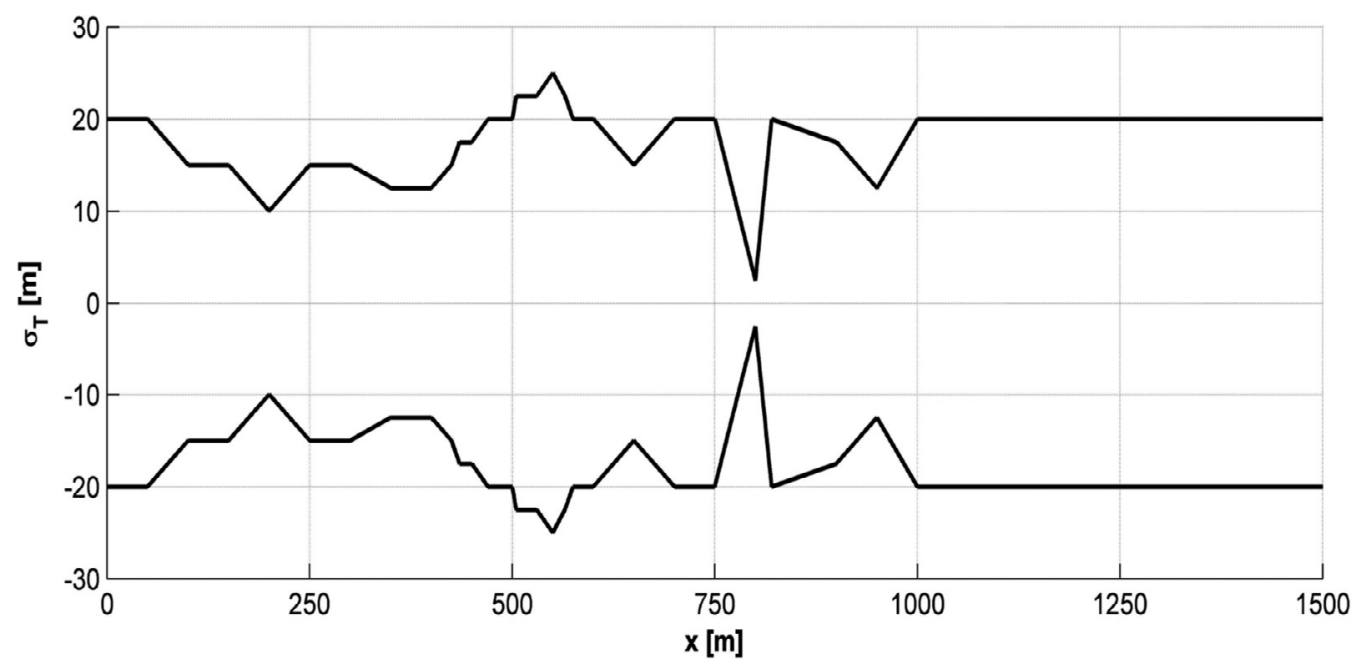

Fig. 2. Channel width for the water at rest test case.
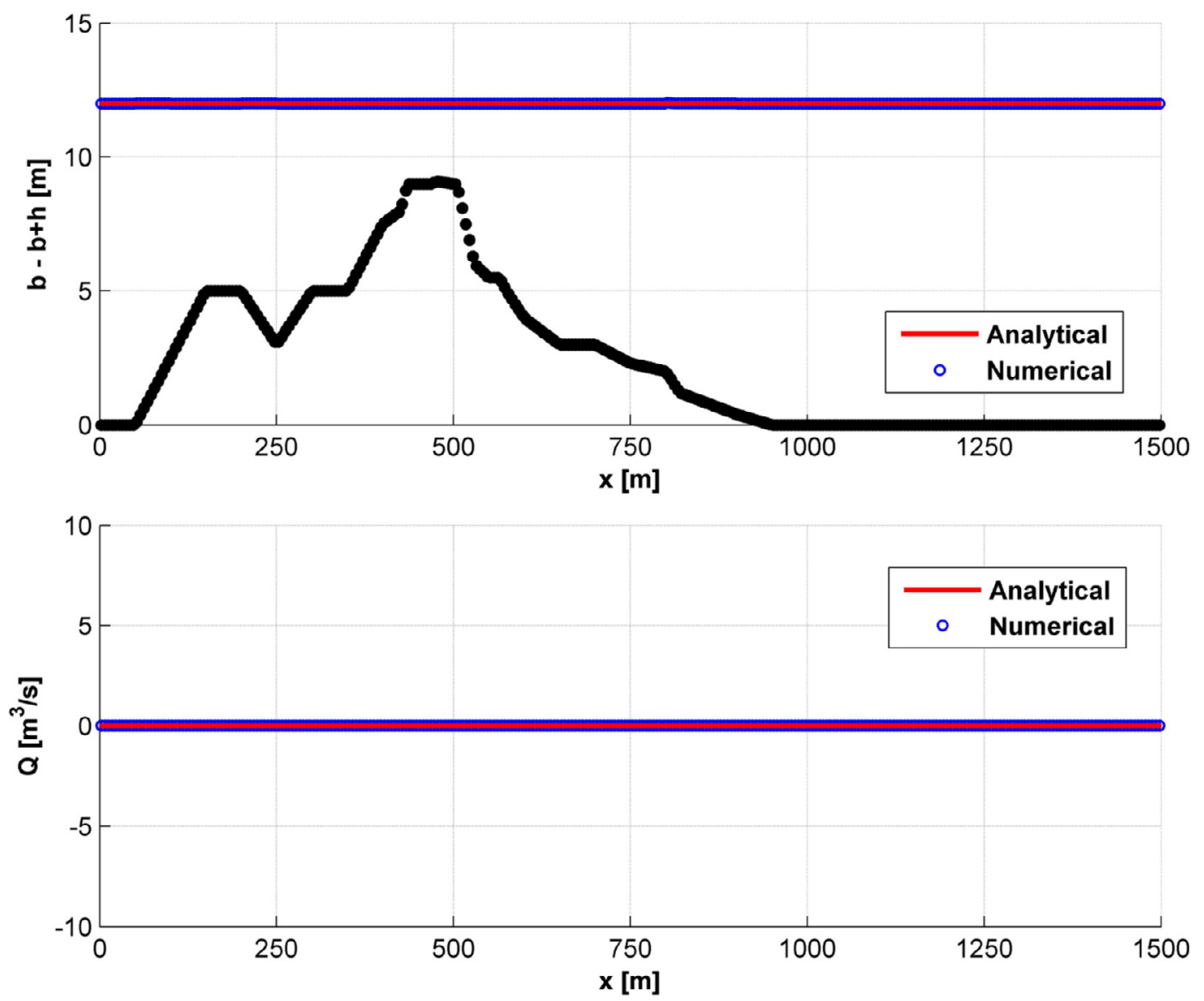

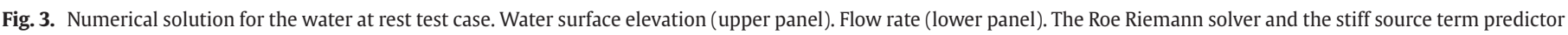
step were used in the simulation.

The DG scheme with TVB limiter, with parameter $K=0.1$ from Eq. (23), provides a less sharp shock profile than the one of the linear DG scheme and a less oscillatory solution.

In the case of a stronger shock (test B) the linear DG scheme crashes and is not able to reproduce the analytical solution. The figure shows the results from the DG scheme with TVB limiter, using $K=0.5$ and $K=0.001$. In the first case the profile is quite irregular, as opposite to the other one where the numerical solution is more regular. In both cases, some numerical diffusivity spreads the numerical solution.

\subsubsection{Ventury-type flume}

The numerical model is now applied for simulating the propagation of a flood wave in a $2024 \mathrm{~m}$ long straight rectangular channel having a bed slope of $0.55 \%$; as in Fig. 5.

Channel width $B_{0}$ is $30 \mathrm{~m}$ except for the central reach where it narrows to a width $B_{c}$. The narrowed central reach is $24 \mathrm{~m}$ long and consists of three $8 \mathrm{~m}$ long sub-reaches. In the first sub-reach, the channel width linearly drops from $B_{0}$ to $B_{c}$, then it remains constant to $B_{c}$, and in the third sub-reach linearly increases back to the full width $B_{0}$. In this test case the contraction coefficient $c=B_{c} / B_{0}=0.2$ is used. 

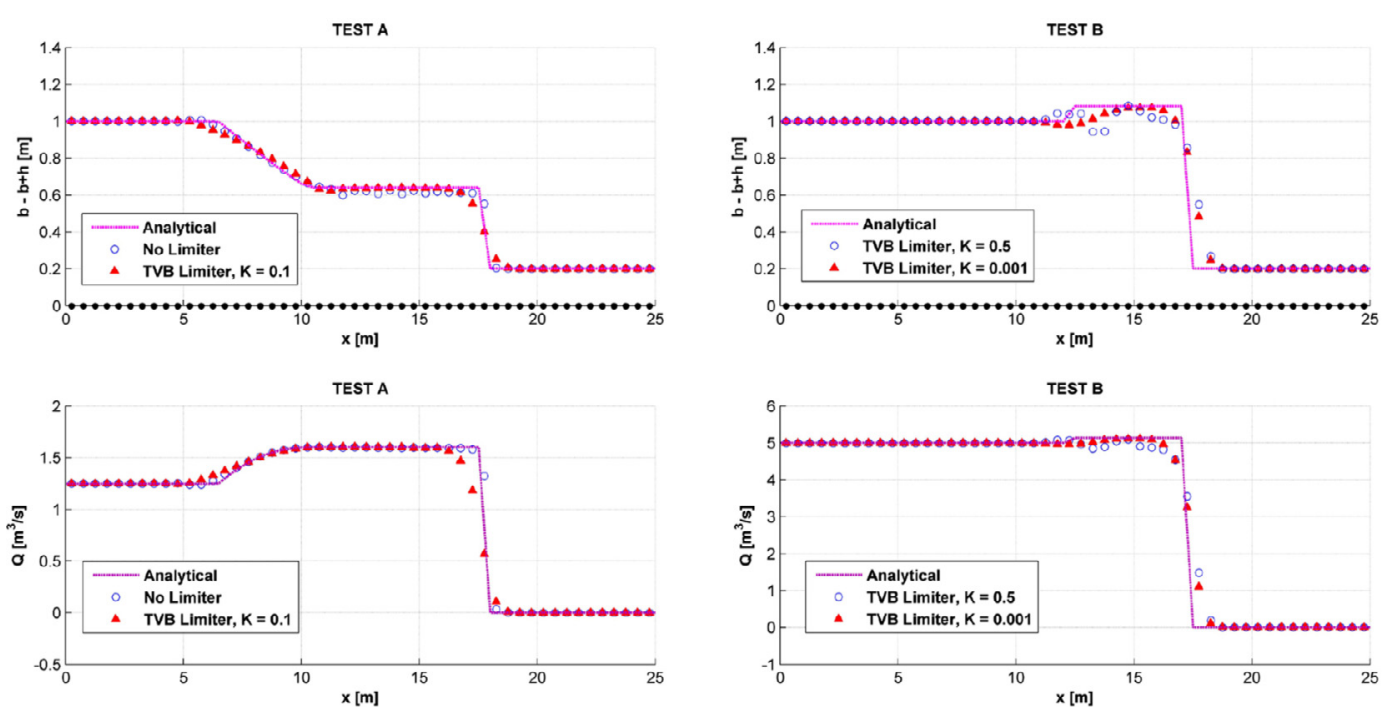

Fig. 4. Numerical solution for test $\mathrm{A}$ at $t=2.0 \mathrm{~s}$ (left column) and for test $\mathrm{B}$ at $t=1.2 \mathrm{~s}$ (right column).
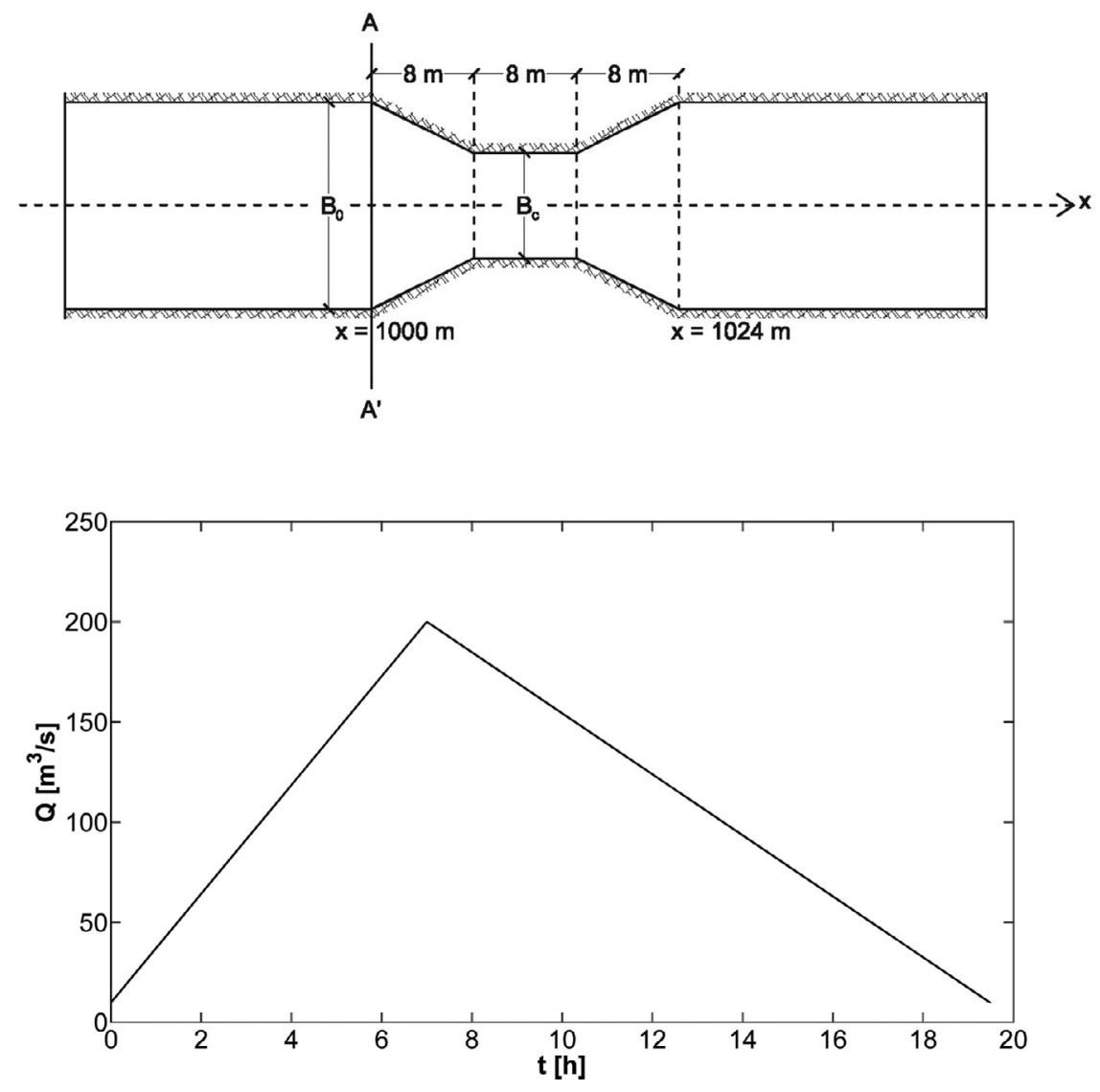

Fig. 5. Sketch and notation (upper panel); input hydrograph (lower panel).

The input flow rate is a triangular hydrograph with a peak discharge of $200 \mathrm{~m}^{3} / \mathrm{s}$, duration of $20 \mathrm{~h}$, discharge growth rate of $27 \mathrm{~m}^{3} / \mathrm{s} / \mathrm{h}$ and discharge decrease rate of $15 \mathrm{~m}^{3} / \mathrm{s} / \mathrm{h}$; see Fig. 5 . The Osher Riemann solver was used and the normal flow depth was enforced downstream.

As far as the grid spacing is concerned, $\Delta x=30 \mathrm{~m}$ when $x \leq$ $900 \mathrm{~m}$, then $\Delta x$ reduces to $20 \mathrm{~m}$ when $900 \mathrm{~m} \leq x \leq 1000 \mathrm{~m}$ and it becomes $8 \mathrm{~m}$ in the contracted reach of channel; the grid spacing is symmetrical with respect to the middle of the channel. Notice that the channel contraction is represented by four cross-sections: two are located just upstream and downstream of the contraction and the other two are at both ends of the narrowest reach. This kind of geometrical representation follows the classical cross-section spacing used in well-known 1D numerical models (such as Hec-Ras, Usace).

The resulting rating curve has been calculated with fixed bed conditions in the cross-section A-A' positioned just upstream the channel contraction at $x=1000 \mathrm{~m}$ (Fig. 5)

The numerical solution is compared to a reference analytical solution obtained modeling the upstream straight reach and the contraction reach as a Venturi flume. For the present test case, no 


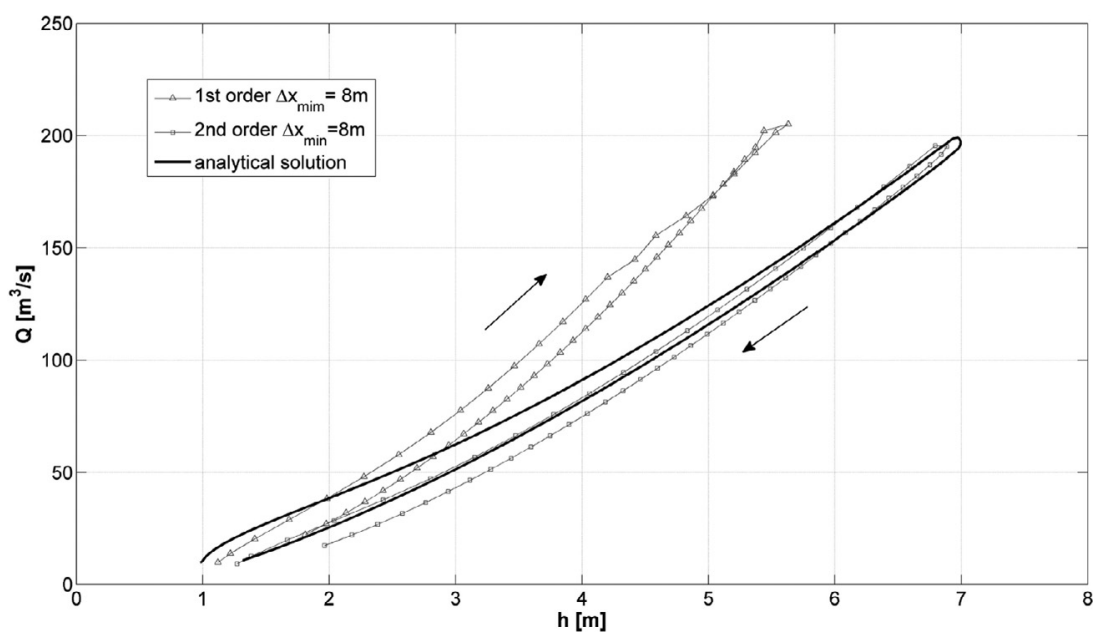

Fig. 6. The rating curves in the cross-section A-A' at 1 st and 2 nd order of accuracy and comparison with the analytical solution (fixed bed condition, $c=0.2$ ).

experimental measurements are available. Water levels in the upstream reach have been calculated using a quasi-static approximation by which their time evolution was driven by the difference between the input flow hydrograph and the output flow in the channel contraction; this approximation appears to be sound as the water levels account for about $90 \%$; of the characteristic speed of waves, being the Froude number around 0.1 in the test case.

The output flow was evaluated assuming the critical flow depth to establish at the contraction.

Results in Fig. 6 show the analytical solution is captured by the 2nd order scheme; the 1st order scheme leads to a relevant overestimation of flow discharge, for a given water level. This finding demonstrates the capability of the present scheme to deal with abrupt geometric changes and how the application of a 1st order scheme may lead to inaccurate results on a coarse grid.

\subsubsection{Subcritical flow in an irregular channel}

The test case is the subcritical flow in a short domain problem, as described by Delestre et al. [17]. It was here reproduced in order to check the order of accuracy of the numerical scheme. The steady state test consists in a $200 \mathrm{~m}$ long rectangular channel, with a bottom width variable in space as follows:

$B_{1}(x)=10-5 \exp \left(-10\left(\frac{x}{200}-\frac{1}{2}\right)^{2}\right)$ for $0 m \leq x \leq L=200 \mathrm{~m}$

The Manning friction coefficient is $n=0.03 \mathrm{~s} / \mathrm{m}^{1 / 3}$. The assumed boundary conditions are: constant flow rate of $Q=20 \mathrm{~m}^{3} / \mathrm{s}$ upstream and constant water depth of $h_{\text {out }}=0.9 \mathrm{~m}$ downstream. The
Table 1

Convergence rate study for the test case of a subcritical flow in an irregular channel.

\begin{tabular}{lllll}
\hline$\Delta x[\mathrm{~m}]$ & $L_{2}$ & $o\left(L_{2}\right)$ & $L_{\text {inf }}$ & $o\left(L_{\text {inf }}\right)$ \\
\hline 4 & $1.78 \cdot 10^{-04}$ & & $9.41 \cdot 10^{-05}$ & \\
2 & $6.02 \cdot 10^{-05}$ & 1.56 & $3.02 \cdot 10^{-05}$ & 1.64 \\
1 & $1.80 \cdot 10^{-05}$ & 1.74 & $8.43 \cdot 10^{-06}$ & 1.84 \\
0.5 & $4.42 \cdot 10^{-06}$ & 2.03 & $2.07 \cdot 10^{-06}$ & 2.02 \\
0.25 & $1.12 \cdot 10^{-06}$ & 1.98 & $5.04 \cdot 10^{-07}$ & 2.04 \\
\hline
\end{tabular}

water depth is calculated as follows:

$h(x)=0.9+0.3 \exp \left(-20\left(\frac{x}{200}-\frac{1}{2}\right)^{2}\right)$

The simulations have been performed decreasing the grid spacing from 4 to $0.25 \mathrm{~m}$ and a convergence rate table has been calculated. The Roe Riemann solver was used in the calculations. The results are shown in Table 1 . The errors are expressed with the $L_{1}$ and $L_{\text {inf }}$ norms of the hydraulic depth $h$. Results indicate the scheme to be 2 nd order accurate.

The water surface profile for the simulation with $\Delta x=2 \mathrm{~m}$ is shown in Fig. 7.

\subsubsection{Propagation of a sediment hump}

We reproduced the movable bed test case, as described by Lyn and Altinakar [18], of the propagation of a sediment hump. The simulated test case is the one termed as "Near Critical, High-Transport Case" in the paper.

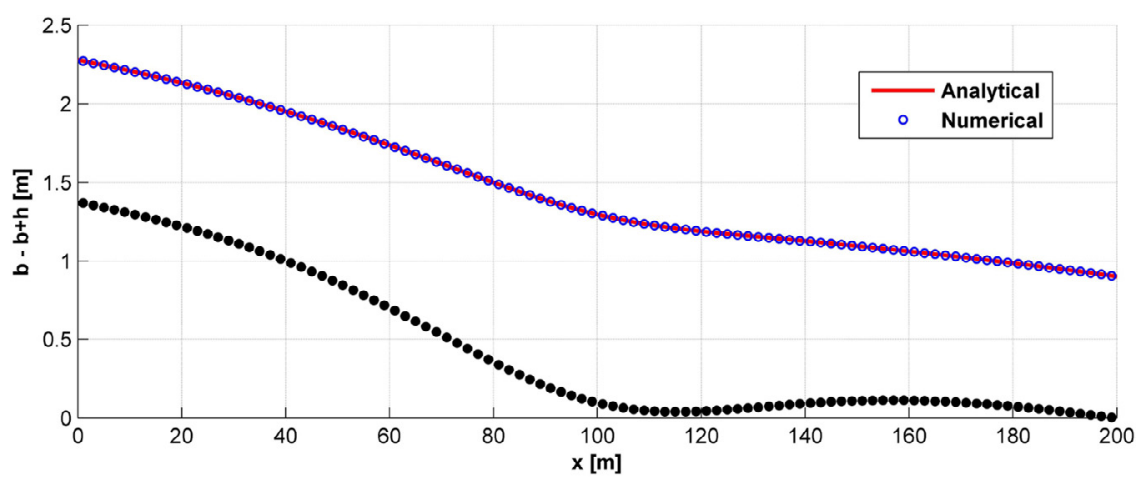

Fig. 7. Numerical solution for the subcritical flow in a short domain problem. Water surface profile for the simulation with $\Delta x=2 \mathrm{~m}$. 

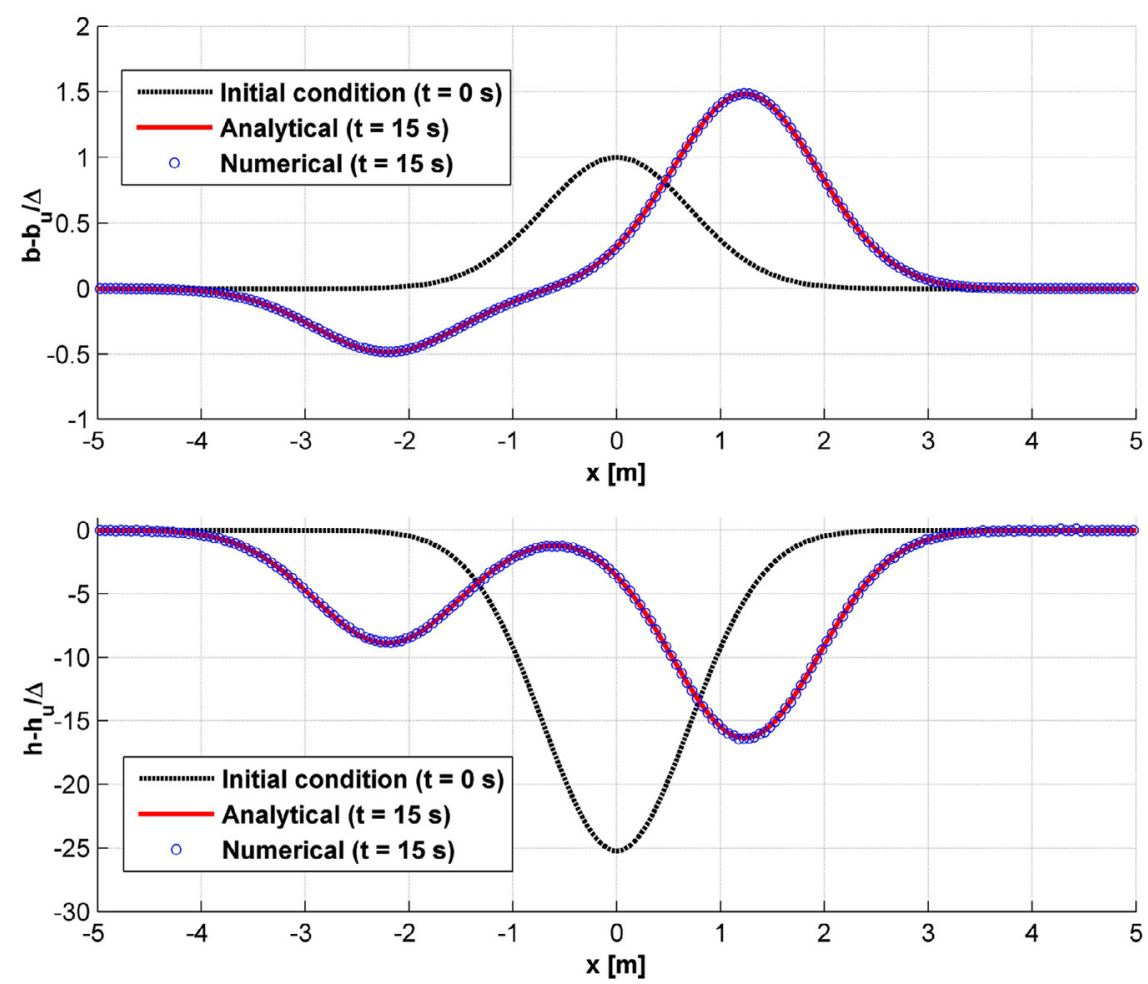

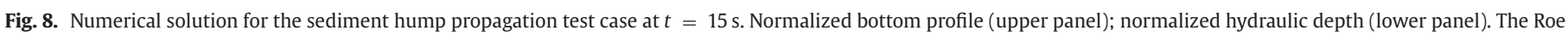
Riemann solver was used in the simulations.

The test case involves the propagation of a small bed disturbance in a channel subject to a uniform flow with Froude number $F_{U}=0.96$, constant hydraulic depth $h_{U}=1.0 \mathrm{~m}$ and thalweg elevation $b_{U}=0.0 \mathrm{~m}$. The cross-section of the channel is rectangular with unit width. The initial disturbance on bottom topography is defined by the following:

$b_{0}(x)=\Delta \cdot e^{-x^{2}}$

The bed perturbation amplitude is chosen to be very low and it is set to $\Delta=10^{-5} \mathrm{~m}$.

The bedload transport equation used is the Grass equation [12]:

$Q_{s}=A_{g} \cdot\left(u-u_{c r}\right)^{m_{g}} \cdot \sigma_{T}$

where $u$ is the flow velocity, $A_{g}$ and $m_{g}$ are calibration parameters used to model the interactions between sediments and flow and $u_{c r}$ is a critical flow velocity for bedload transport initiation. In the simulation, it was chosen $A_{g}=3.4 \times 10^{-4}$ and $m_{g}=2.65$. The volume fraction was chosen to be $\phi=0.6$. Finally, the critical flow velocity $u_{c r}$ was set in order to adjust the nondimensional bedload transport parameter, used in Lyn and Altinakar [18] to linearize the set of equations:

$\psi_{U}=\frac{1}{\phi h_{U}} \cdot \frac{\partial\left(Q_{S} / \sigma_{T}\right)}{\partial u}$

For the present test case it was set $\psi_{U}=2.5 \times 10^{-3}$. The enforced boundary conditions are the ones of the uniform flow away from the sediments hump. The initial condition corresponds to the fixed bed steady flow water surface profile over the hump. The analytical solution is found by linearizing the system of equations about the uniform flow. The grid spacing was set to $\Delta x=0.05 \mathrm{~m}$.

Fig. 8 shows the resulting normalized water surface and bottom profiles after $15 \mathrm{~s}$ of simulation.

The comparison with the analytical solution indicates the capability of our scheme to fully capture the direction and celerity of propagation of bed and water surface disturbances.

\subsubsection{Flood wave in the Ombrone Pistoiese river}

The propagation of a flood wave along a reach of the Ombrone Pistoiese river (Tuscany, Italy) is simulated in the present test case. The simulated river reach is $6 \mathrm{~km}$ long with a bed slope of $0.06 \%$; it includes the gauge station of Poggio a Caiano (Pistoia) installed upstream of a bridge where flow discharge measurements were carriedout. This reach is described through 142 compound crosssections. The average width for the main channel is about $20 \mathrm{~m}$ while the side overbanks are $10 \mathrm{~m}$ wide in average. Also it was taken $D_{50}=0.011 \mathrm{~m}$ from field samples of bed material and Manning coefficient is set equal to $0.036 \mathrm{~s} / \mathrm{m}^{1 / 3}$ for channel and $0.083 \mathrm{~s} / \mathrm{m}^{1 / 3}$ for banks. The Manning coefficients were chosen in order to reproduce the existing steady flow rating curve for the station. The volume fraction was chosen to be $\phi=0.6$.

The following boundary conditions are enforced: at the upstream boundary a 2 year return period hydrograph with a peak discharge of $285 \mathrm{~m}^{3} / \mathrm{s}$, at the downstream boundary the steady flow water levels. Results in Fig. 9 show a comparison between the rating curves obtained from interpolation of field measurements and from the numerical model.

The comparison is encouraging as the numerical scheme captures the interpolated rating curve and the observed data. In particular, the measurement of December 9th, 2006 was taken while the water levels were decreasing, that is during the descending phase of the flow hydrograph; this aspect is reproduced by the numerical scheme. Also, the January 17th, 2008 measurement was taken during the increasing phase of hydrograph; this also is correctly reproduced by the scheme.

\subsection{Computation of rating curves}

Results from test case of Section 4.1.3 are now extended by considering different contractions coefficients $c$ : namely a mild $(c=0.8)$, medium $(c=0.5)$, and strong $(c=0.2)$ contraction ratio. Numerical simulations are carried out with both fixed and movable bed 


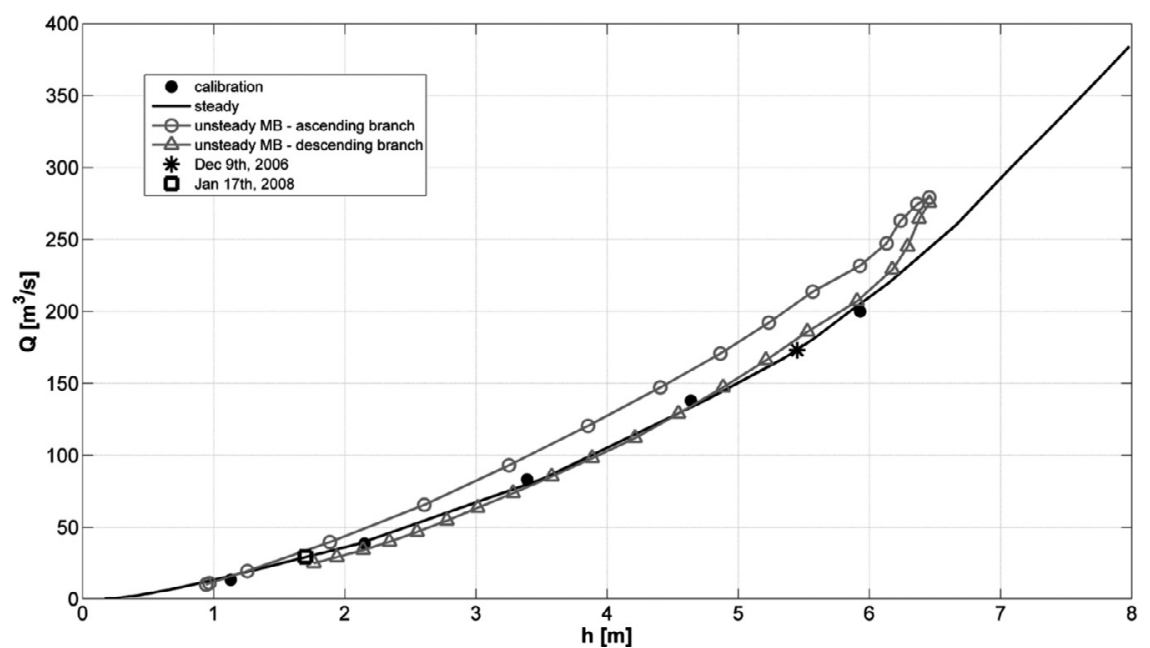

Fig. 9. The rating curves in steady flow condition (black curve) and unsteady flow condition in movable bed (grey curve) in comparison with data from calibration (black points) and field measurements (black square and star) at the gauging station in Poggio a Caiano (Pistoia, Italy).
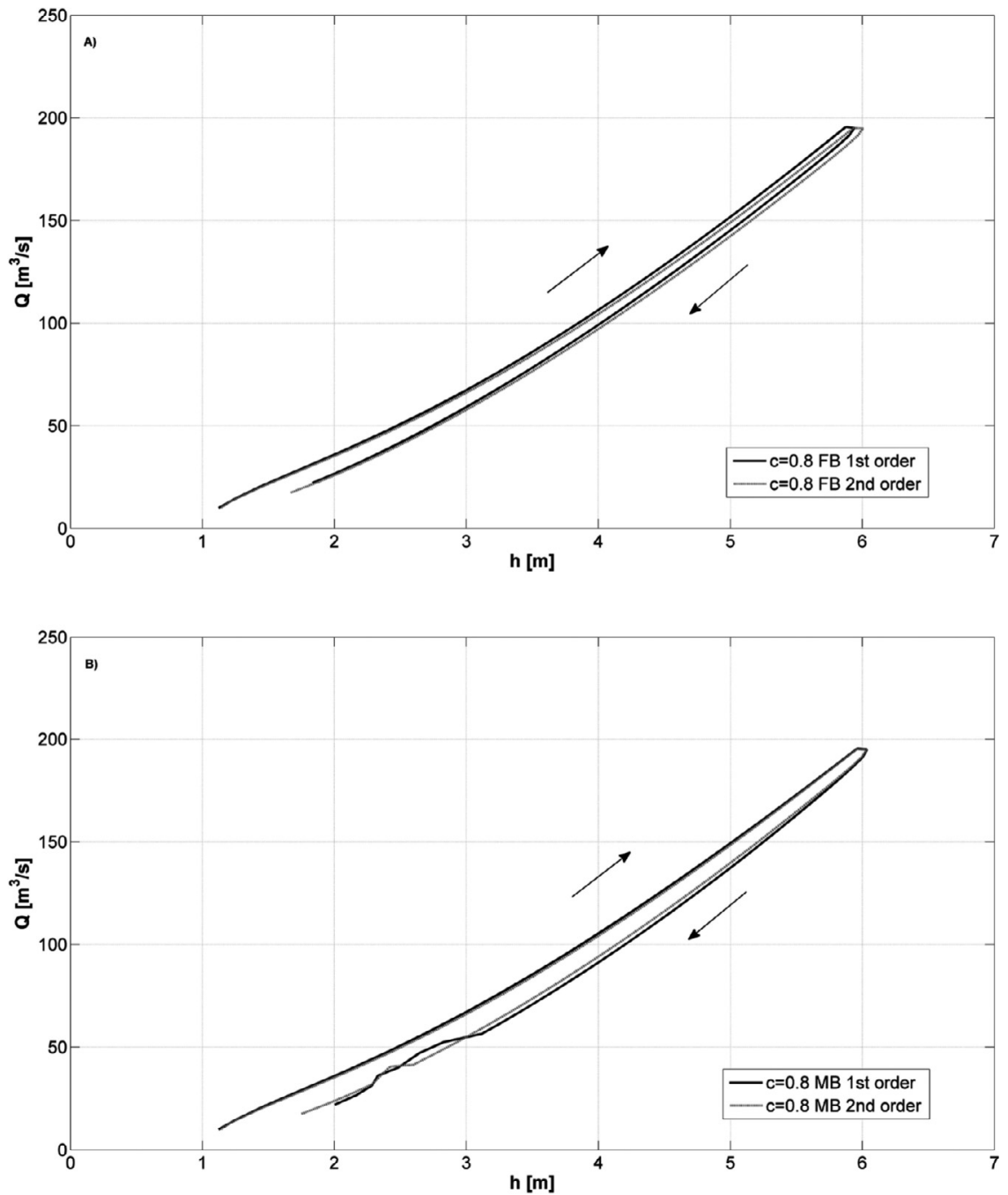

Fig. 10. The rating curves in fixed (upper panel) and movable (lower panel) bed conditions in the cross-section A-A' when $c=0.8$. The 2 nd order simulation was performed with the moderately stiff source term predictor step. 

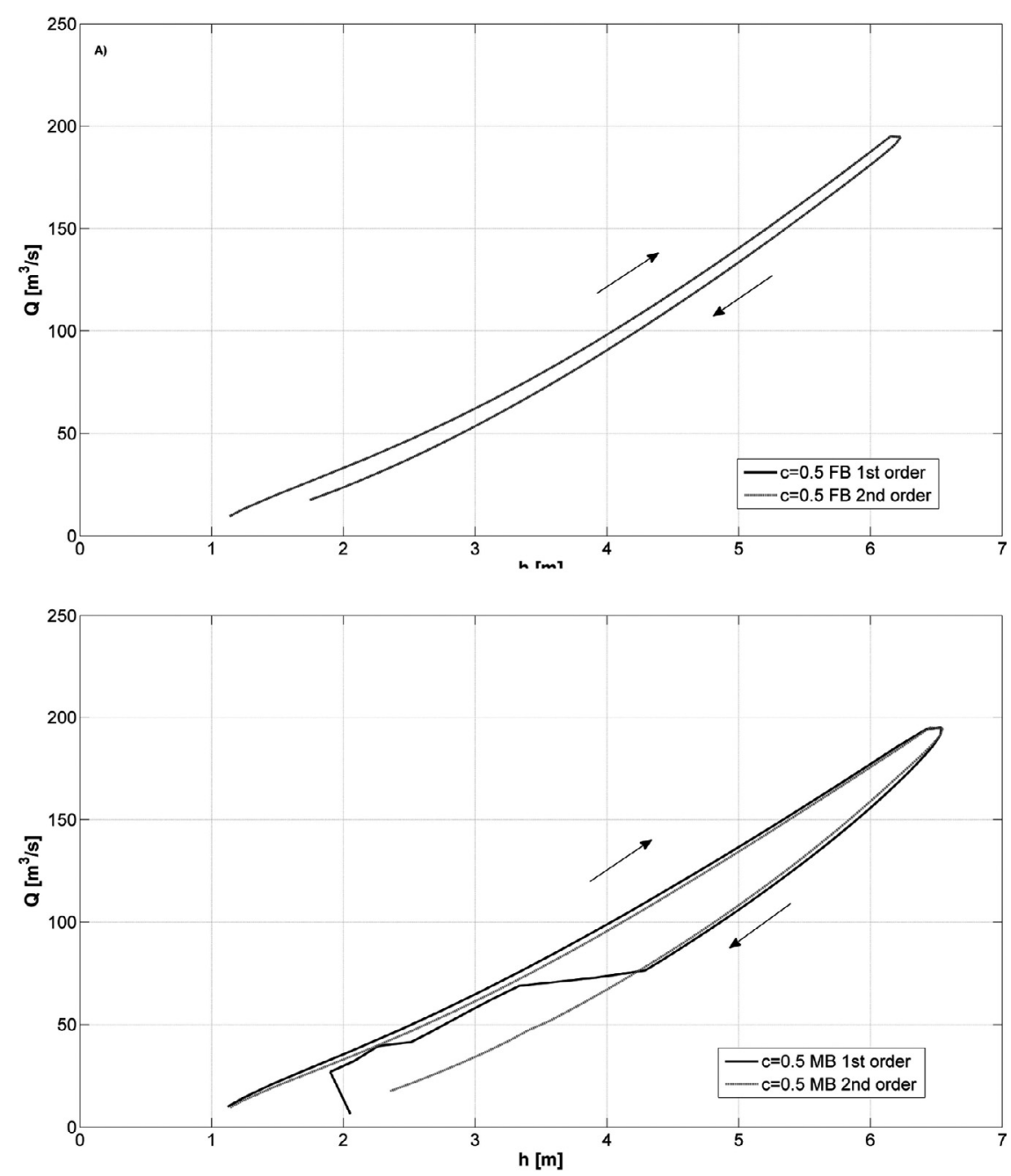

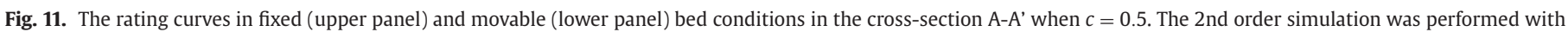
the moderately stiff source term predictor step.

conditions. In the latter case, the total sediment load formula by Ackers and White [29] is adopted with $D_{35}=0.002 \mathrm{~m}$. The volume fraction was chosen to be $\phi=0.6$. The inflow hydrograph is the same used in Section 4.1.3. Moreover, results are also compared with the 1 st order version of the present scheme.

As in Section 4.1.3, rating curves have been calculated in the crosssection A-A' (see Fig. 5), where they are not affected by the presence of the hydraulic jump that might establish after the channel contraction; this is to replicate what can be commonly observed in river monitoring stations, where water level gauges are typically located on bridges upstream of width narrowing.

Fig. 10 shows the rating curves in the case of the mild contraction ratio $(c=0.8)$. It appears that the results at the 2 nd order overlap with the ones at the 1st order. This finding is confirmed in the case of movable bed; a slight deviation can only be detected in the falling limb at lower discharges.

In the case of the medium contraction coefficient $c=0.5$, results in Fig. 11 show that two schemes provide identical rating curves when the bed is fixed; however, in the case of movable bed, a deviation occurs during the falling limb for discharges lower than about $75 \mathrm{~m}^{3} / \mathrm{s}$. The scheme at the 1 st order of accuracy predicts a sudden reduction of flow levels and the classical loop shape of the rating curve almost disappears. Interestingly, the movable character of the bed and in particular the local scour at the channel constriction, enhances the hysteretic behavior of rating curves as the difference in the rising and falling limbs becomes more pronounced.

When a strong contraction is considered $(c=0.2)$, the rating curves predicted by the two schemes considerably deviate both in fixed and movable bed conditions; see Fig. 12. In particular, in fixed bed conditions, for a given water level, the 1 st order scheme greatly overestimates flow discharges of about $40 \%$. Also, the rating curve with the 1 st order scheme appears to have a rather regular shape, therefore it is not possible to detect this misleading result just by visual inspection, without a comparison with the results at the 2nd order of accuracy. In movable bed conditions (Fig. 12), the rating curve at 1 st order attains a rather irregular behavior with the falling limb crossing the rising limb due to a sudden increase of flow discharge together with a reduction of water level.

\section{Discussion and conclusions}

In the present work a 1D model based on a higher order numerical scheme for simulating flood wave propagation on a natural channel with a movable bed has been presented. An important feature of the scheme is represented by its ability at treating the moderately stiff or stiff source terms that may appear in the governing equations due to the occurrence of abrupt changes in the channel geometry.

After the validation of the scheme, its application to the computation of flow rating curves in a schematic channel with a 

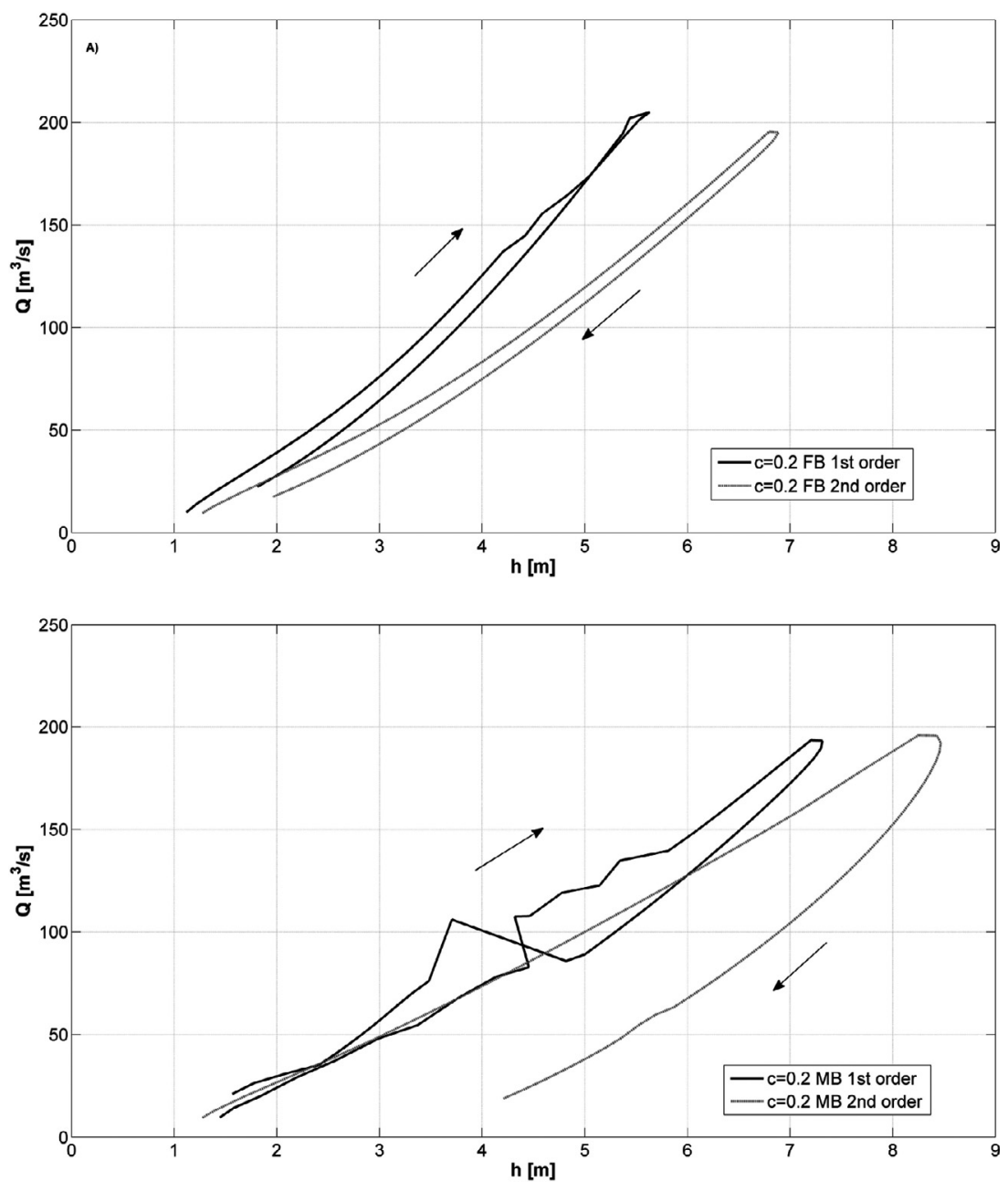

Fig. 12. The rating curves in movable bed conditions in the cross-section A-A' when $c=0.2$. The 2 nd order simulation was performed with the stiff source term predictor step.

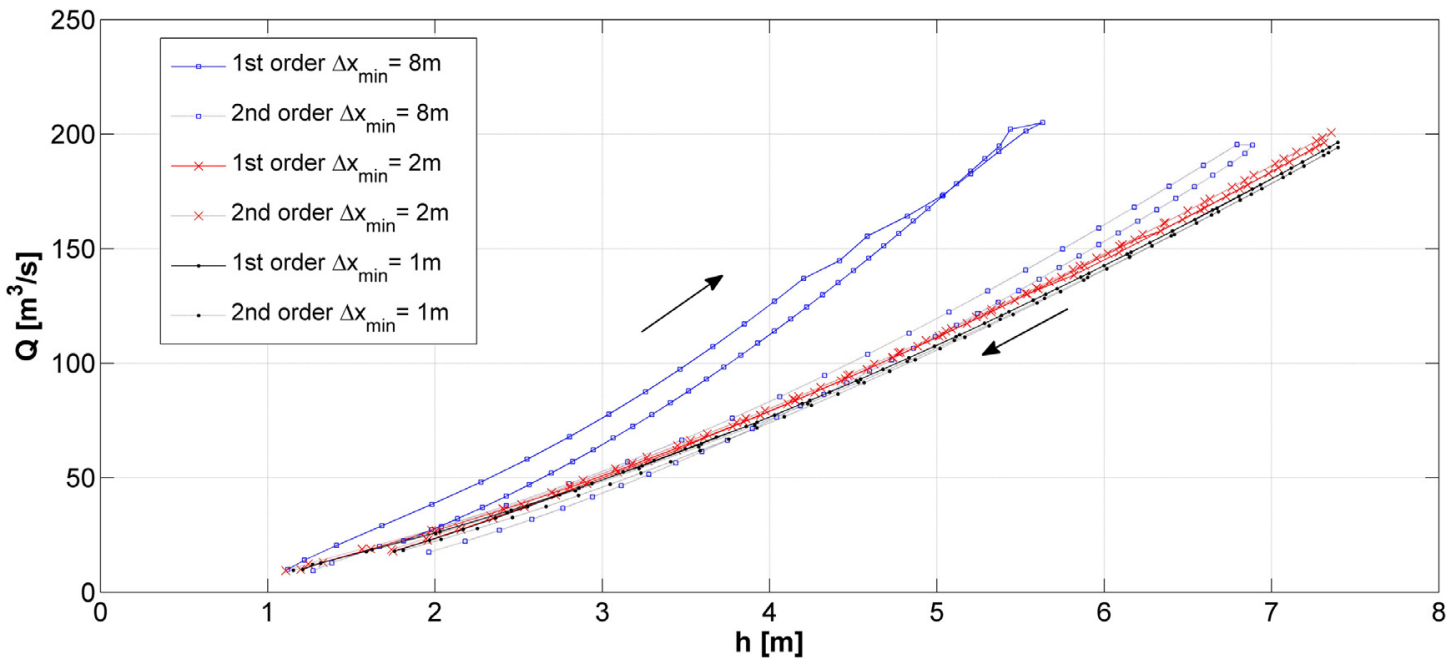

Fig. 13. Rating curves predicted by 1 st and 2 nd order schemes in case of different cross-section spacing and in fixed bed conditions, $c=0.2$.

contraction was presented. The used configuration mimics what can be typically found in rivers, where water level gauges are installed on bridges. In these conditions, rating curves can be directly influenced by the channel narrowing since backwater effects and transition through the critical state may occur.
Simulations were shown considering both fixed and movable bed conditions and using the present 2 nd order numerical scheme and its 1 st order version. The channel contraction was specified using classical geometrical representation by means of four cross-sections, with a constant spacing of $8 \mathrm{~m}$. Results show that, with the chosen grid spacing, rating curves are correctly reproduced only when the 2nd 
order scheme with source terms treatment is employed; a 1st order scheme leads to erroneous results greatly overestimating flow discharge for the given water level.

Much smaller grid spacings of $2 \mathrm{~m}$ and $1 \mathrm{~m}$ in the contracted reach are now considered. Results in Fig. 13 show a comparison between the rating curves considering the different spacings both at the first and the second order of accuracy. It appears that results with 1 st order scheme are crucially dependent on grid-spacing while this dependence is almost negligible at the 2nd order of accuracy. In the case of the tight spacing of $2 \mathrm{~m}$, and more strongly in the case of $1 \mathrm{~m}$, the $1 \mathrm{st}$ and the 2 nd order schemes provide similar results.

This finding is not surprising as 1 st order schemes might suffer from an inaccurate representation of source terms. The fact that the results from the 1 st order scheme overlap with the ones from the 2nd order scheme, only when grid spacing is drastically reduced, emphasizes the issue of the proper geometrical channel representation in terms of cross-section spacing in 1D models. Classical representation of channel obstructions by means of four cross-sections may lead to inaccurate results and misleading rating curves with 1 st order schemes. On the other hand, 2 nd order schemes are capable of reproducing robust rating curves which are less affected by cross-sections spacing.

Moreover, if the common four cross-sections representation is used, results suggest that rating curves should be computed by using more robust higher-order numerical schemes including special treatment for moderately stiff or stiff source terms.

\section{Acknowledgments}

The authors are grateful to the Tuscany regional authority "Centro Funzionale di Monitoraggio Meteo Idrologico - Idraulico, Servizio Idrologico Regionale" for having contributed to the present research by providing the Ombrone river data used in the present work. Part of such data is not of public domain and was provided to the University of Florence under specific and broader agreements. The Editor Dr. Andrea Rinaldo, the Guest Editor Dr. Annunziato Siviglia and two anonymous referees are acknowledged for having helped improving the presentation of this work by providing useful comments.

\section{Appendix A}

Modal bases functions are orthonormal bases for the space of polynomials either on the normalized space element $[0,1]$ or spacetime element $[0,1] \times[0,1]$ according to the accuracy of the scheme.

The following table shows linear and quadratic space and spacetime bases functions. Expressions of the functions can be obtained starting from a generic set of bases and using the Gram-Schmidt orthonormalization process (see Hoffman and Kunze [30]).

Finally, expression for the matrices needed for the higher order version of the scheme are shown for $N=3$ (2nd order accurate

Table A1

Space and space-time modal bases expression for schemes with up to 3rd order accuracy.

\begin{tabular}{llll}
\hline$N_{0}$ & $N$ & $\phi(\xi)$ & $\theta(\xi, \tau)$ \\
\hline 2 & 3 & $\phi_{1}=1$ & $\theta_{1}=1$ \\
& & $\phi_{2}=\sqrt{3} \cdot(2 \xi-1)$ & $\theta_{2}=\sqrt{3} \cdot(2 \xi-1)$ \\
& & & $\theta_{3}=\sqrt{3} \cdot(2 \tau-1)$ \\
3 & \multirow{2}{*}{6} & $\phi_{1}=1$ & $\theta_{1}=1$ \\
& $\phi_{2}=\sqrt{3} \cdot(2 \xi-1)$ & $\theta_{2}=\sqrt{3} \cdot(2 \xi-1)$ \\
& $\phi_{3}=\sqrt{5} \cdot\left(6 \xi^{2}-6 \xi+1\right)$ & $\theta_{3}=\sqrt{5} \cdot\left(6 \xi^{2}-6 \xi+1\right)$ \\
& & $\theta_{4}=\sqrt{3} \cdot(2 \tau-1)$ \\
& & $\theta_{5}=3-6 \xi-6 \tau+12 \xi \tau$ \\
& & $\theta_{6}=\sqrt{5} \cdot\left(6 \tau^{2}-6 \tau+1\right)$ \\
\hline
\end{tabular}

scheme) and for the bases functions of Table A.1:

$K_{k l}^{\tau}=\left(\begin{array}{ccc}0 & 0 & 2 \sqrt{3} \\ 0 & 0 & 0 \\ 0 & 0 & 0\end{array}\right)$

$M_{k l}=I_{3}$

with $I_{N}$ being the identity matrix of order $N$.

$M_{k l}^{(1)}=\left(\begin{array}{ccc}1 & 0 & \sqrt{3} \\ 0 & 1 & 0 \\ \sqrt{3} & 0 & 1\end{array}\right)$

$M_{k l}^{(0)}=\left(\begin{array}{cc}1 & 0 \\ 0 & 1 \\ -\sqrt{3} & 0\end{array}\right)$

\section{Appendix B}

\section{Predictor step for non stiff or moderately stiff source terms}

Eq. (33) is specialized for a 2 nd order accurate scheme, with the bases indicated in Table A.1 and for the model equations introduced in Section 2 for which $\omega=x$.

In this case, the initial condition of Eq. (36) provides:

$\left\{\begin{array}{l}q_{1, i}-\sqrt{3} q_{3, i}=U_{1, i}^{n} \\ q_{2, i}=U_{2, i}^{n} \\ \mu_{1, i}=\frac{x_{i-1 / 2}+x_{i+1 / 2}}{2} \\ \mu_{2, i}=\frac{\sqrt{3}}{6} \Delta x_{i}\end{array}\right.$

As far as the pure time mode $\widetilde{q}_{3}$ is concerned, the following equations are obtained from Eq. (33):

$\left\{\begin{array}{l}q_{3, i}+\frac{\Delta t}{\Delta x_{i}} \iint_{\dot{\epsilon}_{i}} A\left(\tilde{W}_{h, i}\right) \cdot q_{2, i} d \xi d \tau- \\ \frac{\sqrt{3} \Delta t}{6} \iint_{\dot{\epsilon}_{i}}(S-V)\left(\tilde{W}_{h, i}\right) d \xi d \tau=0 \\ \mu_{3, i}=0\end{array}\right.$

The first equation in B.2 is a nonlinear equation in $q_{k, i}, k=1 . .3$. With simple algebraic manipulations, using also Eq. B.1, it can be cast in the form $q_{3, i}=\eta_{0}+f\left(q_{3, i}\right)$, with $\eta_{0}$ being a known term. It can be solved through the following iteration scheme, with $q_{3, i}^{k}$ indicating the solution at the $k$-th iteration:

1. Check if $q_{3, i}^{(k)}$ solves the equation with a reasonable error. If it does, then halt iterations. Go to step 2 otherwise.

2. Evaluate $q_{3, i}^{(k+1)}=\eta_{0}+f\left(q_{3, i}^{(k)}\right)$.

3. Increase iteration counter $k$ and go to step 1 .

The stationary solution $q_{3, i}^{(0)}=0$ may be chosen as initial guess for the iterations.

\section{Predictor step for stiff source terms}

Eq. (38) is specialized for a 2nd order accurate scheme, with the bases indicated in Table A.1 and for the model equations introduced 
in Section 2 for which $\omega=x$. The following system of nonlinear algebraic equations is obtained:

$$
\left\{\begin{array}{l}
q_{1, i}+\sqrt{3} q_{3, i}-U_{1, i}^{n}+\frac{\Delta t}{\Delta x_{i}} \iint_{\dot{\epsilon}_{i}} 2 \sqrt{3} A\left(\tilde{W}_{h, i}\right) \cdot q_{2, i} d \xi d \tau \\
-\Delta t \iint_{\dot{\epsilon}_{i}}(S-V)\left(\tilde{W}_{h, i}\right) d \xi d \tau=0 \\
q_{2, i}-U_{2, i}^{n}+\frac{\Delta t}{\Delta x_{i}} \iint_{\dot{\epsilon}_{i}} \sqrt{3}(2 \xi-1) \cdot\left(2 \sqrt{3} A\left(\tilde{W}_{h, i}\right) \cdot q_{2, i}\right) d \xi d \tau \\
-\Delta t \iint_{\dot{\epsilon}_{i}} \sqrt{3}(2 \xi-1) \cdot(S-V)\left(\tilde{W}_{h, i}\right) d \xi d \tau=0 \\
q_{3, i}-\sqrt{3} q_{1, i}+\sqrt{3} U_{1, i}^{n} \\
+\frac{\Delta t}{\Delta x_{i}} \iint_{\dot{\epsilon}_{i}} \sqrt{3}(2 \tau-1) \cdot\left(2 \sqrt{3} A\left(\tilde{W}_{h, i}\right) \cdot q_{2, i}\right) d \xi d \tau \\
-\Delta t \iint_{\dot{\epsilon}_{i}} \sqrt{3}(2 \tau-1) \cdot(S-V)\left(\tilde{W}_{h, i}\right) d \xi d \tau=0
\end{array}\right.
$$

Eqs. B.3 can't be solved by using an iterative scheme such as the one used for the non stiff source term case. A more sophisticated technique, like the inexact Newton method algorithm with backtracking proposed by Pernice and Walker [31], should instead be used.

\section{Corrector step}

Eq. (54) is specialized for a 2nd order accurate scheme, with the bases indicated in Table A.1 and for the model equations introduced in Section 2 for which $\omega=x$. Note that in this case the geometry function $\omega$ is a linear continuous function and the $(D+1)$-th component of the predicted state $\mu_{h, i}$ can exactly be reproduced starting from a 2nd order scheme. Therefore $\mu_{h, i}$ does not show discontinuities at interfaces and the $I_{i+1 / 2}^{ \pm}$terms disappear in Eq. (54). The resulting scheme is as follows:

$\left\{\begin{array}{l}U_{1, i}^{n+1}=U_{1, i}^{n}-\frac{\Delta t}{\Delta x_{i}}\left(\mathcal{H}_{i-1 / 2}^{+}+\mathcal{H}_{i+1 / 2}^{-}+\mathcal{L}_{1, i}\right) \\ U_{2, i}^{n+1}=U_{2, i}^{n}+\frac{\Delta t}{\Delta x_{i}}\left(\sqrt{3} \mathcal{H}_{i-1 / 2}^{+}-\sqrt{3} \mathcal{H}_{i+1 / 2}^{-}-\mathcal{L}_{2, i}\right)\end{array}\right.$

\section{References}

[1] Dumbser M, Castro M, Pares C, Toro E. Ader schemes on unstructured meshes for nonconservative hyperbolic systems: applications to geophysical flows. Comput Fluids 2009;38(9):1731-48. http://dx.doi.org/10.1016/j.compfluid.2009.03.008.

[2] Toro EF. Riemann solvers and numerical methods for fluid dynamics. Springer; 2009.

[3] Dumbser M, Enaux C, Toro E . Finite volume schemes of very high order of accuracy for stiff hyperbolic balance laws. J Comput Phys 2008a;227(8):3971-4001. http://dx.doi.org/10.1016/j.jcp.2007.12.005.

[4] Catella M, Paris E, Solari L. Conservative scheme for numerical modelling of flow in natural geoemtry. J Hydraul Eng 2008;134(6):736-48. http://dx.doi.org/10.1061/(ASCE)0733-9429(2008)134:6(736).

[5] Audusse E, Bouchut F, Bristeau M, Klein R, Perthame B. A fast and stable wellbalanced scheme with hydrostatic reconstruction for shallow water flows. SIAM J Sci Comput 2004;25(6):2050-65. http://dx.doi.org/10.1137/S1064827503431090.

[6] Caleffi V, Valiani A, Bernini A. High-order balanced cweno scheme for movable bed shallow water equations. Adv Water Resour 2007;30(4):730-41. http://dx.doi.org/10.1016/j.advwatres.2006.06.003.
[7] Siviglia A, Nobile G, Colombini M. Quasi-conservative formulation of the onedimensional Saint-Venantexner model. J Hydraul Eng 2008;134(10):1521-6. http://dx.doi.org/10.1061/(ASCE)0733-9429(2008)134:10(1521).

[8] Canestrelli A, Siviglia A, Dumbser M, Toro E. Well-balanced high-order centered schemes for non-conservative hyperbolic systems. Applications to shallow water equations with fixed and mobile bed. Adv Water Resour 2009;32(6):834-44 http://dx.doi.org/10.1016/j.advwatres.2009.02.006.

[9] Schmidt A, Yen B. Theoretical development of stage-discharge ratings for subcritical open-channel flows. J Hydraul Eng 2008;134(9):1245-56 http://dx.doi.org/10.1061/(ASCE)0733-9429(2008)134:9(1245).

[10] Francalanci S, Paris E, Solari L. A combined field sampling-modeling approach for computing sediment transport during flash floods in a gravel-bed stream. Water Resour Res 2013;49(10):6642-55. http://dx.doi.org/10.1002/wrcr.20544.

[11] Chow V. Open-channel hydraulics. McGraw-Hill; 1959.

[12] Grass A. Sediment transport by waves and currents. Tech rep, SERC London Cent Mar Technology report no. FL29 1981.

[13] Castro MJ, Fernandez-Nieto ED, Ferreiro M. Sediment transport models in shallow water equations and numerical approach by high order finite volume methods. Comput Fluids 2008;37(3):299-316. http://dx.doi.org/10.1016/j.compfluid.2007.07.017.

[14] Cordier S, Le M, De Luna TM. Bedload transport in shallow water models: Why splitting (may) fail. How hyperbolicity (can) help, Adv Water Resour 2011;34(8):980-9. http://dx.doi.org/10.1016/j.advwatres.2011.05.002.

[15] Titarev V, Toro E. Ader schemes for three-dimensional nonlinear hyperbolic systems. J Comput Phys 2005;204(2):715-36. http://dx.doi.org/10.1016/j.jcp.2004.10.028.

[16] Dumbser M, Balsara D, Toro E, Munz C . A unified framework for the construction of one-step finite volume and discontinuous Galerkin schemes on unstructured meshes. J Comput Phys 2008b;227(18):8209-53. http://dx.doi.org/10.1016/j.jcp.2008.05.025.

[17] Delestre O, Lucas C, Ksinant PA, Darboux F, Laguerre C, Vo TNT, et al Swashes: a compilation of shallow water analytic solutions for hydraulic and environmental studies. Int J Numer Methods Fluids 2013;72(3):269-300. http://dx.doi.org/10.1002/fld.3741.

[18] Lyn D, Altinakar M. St. Venant-Exner equations for near-critical and transcritical flows. J Hydraul Eng 2002;128(6):579-87. http://dx.doi.org/10.1061/(ASCE)07339429(2002)128:6(579).

[19] Cockburn B, Shu C. Tvb Runge-Kutta local projection discontinuous Galerkin finite element method for conservation laws. ii. General framework. Math Comput 1989;52:411-35. http://dx.doi.org/10.1090/S0025-5718-1989-0983311-4.

[20] Cockburn B, Lin S, Shu C. Tvb Runge-Kutta local projection discontinuous Galerkin finite element method for conservation laws iii: One-dimensional systems. J Comput Phys 1989;84(1):90-113. http://dx.doi.org/10.1016/0021-9991(89)90183-6.

[21] Atkins H, Shu C. Quadrature-free implementation of discontinuous Galerkin method for hyperbolic equations. AIAA J 1998;36(5):775-82. http://dx.doi.org/10.2514/2.436

[22] Pares C, Castro M. On the well-balance property of Roe's method for nonconservative hyperbolic systems. Applications to shallow-water systems. ESAIM: Math Model Numer Anal 2004:38:821-52. http://dx.doi.org/10.1051/m2an:2004041.

[23] Roe P. Approximate Riemann solvers, parameter vectors, and difference schemes. J Comput Phys 1981;43(2):357-72. http://dx.doi.org/10.1016/00219991(81)90128-5.

[24] Harten A, Hyman J. Self adjusting grid methods for one-dimensional hyperbolic conservation laws. J Comput Phys 1983;50(2):235-69. http://dx.doi.org/10.1016/0021-9991(83)90066-9.

[25] Engquist B, Osher S. One-sided difference approximations for nonlinear conservation laws. Math Comput 1981;36:321-51. http://dx.doi.org/10.1090/S0025-57181981-0606500-X.

[26] Dumbser M, Toro E. A simple extension of the Osher Riemann solver tonon-conservative hyperbolic systems. J Sci Comput 2011;48(1-3):70-88. http://dx.doi.org/10.1007/s10915-010-9400-3.

[27] Jiang G, Shu C. On a cell entropy inequality for discontinuous Galerkin methods. Math Comput 1994;62:531-8. http://dx.doi.org/10.1090/S0025-5718-19941223232-7.

[28] Garcia-Navarro P, Vazquez-Cendon ME. On numerical treatment of the source terms in the shallow water equations. Comput Fluids 2000;29(8):951-79. http://dx.doi.org/10.1016/S0045-7930(99)00038-9.

[29] Ackers P, White R. Sediment transport: new approach and analysis. J Hydraul Div 1973;99(HY11):2041-60.

[30] Hoffman K, Kunze R. Linear algebra. Englewood Cliffs, New Jersey: Prentice - Hall 1971.

[31] Pernice M, Walker H. Nitsol: a newton iterative solver for nonlinear systems. SIAM J Sci Comput 1998;19(1):302-18. http://dx.doi.org/10.1137/S1064827596303843. 